TRANSACTIONS OF THE

AMERICAN MATHEMATICAL SOCIETY

Volume 357, Number 9, Pages 3623-3653

S 0002-9947(04)03597-4

Article electronically published on December 9, 2004

\title{
SUSPENSIONS OF CROSSED AND QUADRATIC COMPLEXES, CO-H-STRUCTURES AND APPLICATIONS
}

\author{
FERNANDO MURO
}

\begin{abstract}
Crossed and quadratic modules are algebraic models of the 2-type and the 3-type of a space, respectively. In this paper we compute a purely algebraic suspension functor from crossed to quadratic modules which sends a 2-type to the 3-type of its suspension. We also give some applications in homotopy theory and group theory.
\end{abstract}

\section{INTRODUCTION}

Crossed modules were introduced by J. H. C. Whitehead ([23]) in order to give an algebraic invariant of a $C W$-complex stronger than the cellular chain complex with local coefficients. Almost at the same time Mac Lane ([18]) used them to describe the third cohomology of a group; moreover, in [19] Whitehead and Mac Lane proved that crossed modules are algebraic models of the 2-type of a $C W$-complex. Although they are defined by non-abelian groups, crossed modules have Peiffer nilpotency degree 1 (see [22] and [9]), hence they are in some sense abelian-like objects. Their non-abelian analogues in the sense of Peiffer are termed precrossed modules.

In 7] Baues introduced quadratic modules to describe the 3-type of a $C W$ complex. They are defined by means of non-abelian groups as well, but have Peiffer nilpotency degree 2. Previously Conduché had defined similar objects called 2crossed modules and showed their relation with the fourth cohomology of a group (16]). 2-crossed modules have been extensively studied and generalized to other contexts; see for example [21] and [3. However, despite their name, they are generalizations of precrossed modules rather than crossed modules, because they do not satisfy any Peiffer nilpotency condition.

Whitehead's homotopy systems [23, now usually called crossed complexes, are chain complexes of groups which in low dimensions have a crossed module and in higher dimensions consist of modules over a group. Baues constructs quadratic complexes in a similar way by using quadratic modules, and proves that the categories of crossed and quadratic complexes are homotopy categories ([7]). In fact, they are $I$-categories in the sense of [6] when we restrict to totally free objects, which are the cofibrant models. A Quillen model category structure was already known for crossed complexes ([14]).

Received by the editors February 19, 2003 and, in revised form, December 30, 2003.

2000 Mathematics Subject Classification. Primary 55P40, 55P45, 55P65; Secondary 55U15, 20F14, 20F18, $20 \mathrm{~J} 05$.

The author was partially supported by the MCyT grant BFM2001-3195-C02-01 and the MECD FPU fellowship AP2000-3330. 
When we work with simply connected $C W$-complexes the notion of Peiffer nilpotency corresponds to the usual notion of nilpotency of a group. For example, the 2-type of such a space is described by an abelian group, namely the second homology group. Reduced quadratic modules are a special kind of quadratic modules, describing the 3 -types of simply connected $C W$-complexes (see [7), which are constructed with groups of nilpotency degree 2 .

In this paper we define a suspension functor from crossed modules to reduced quadratic modules (Proposition 3.3. ) that, topologically speaking, sends a 2-type to the 3-type of its suspension (Corollary 4.8). This functor is extended to crossed and quadratic complexes (Proposition 4.1). A notion of suspension exists in the categories of totally free crossed and quadratic complexes, because they are $I$ categories. However this categorical suspension applied to a crossed complex gives simply a chain complex of abelian groups. It does not encode the 3-type of the suspension, but only the 2-type. The functor constructed here avoids this problem. Furthermore, we prove that the suspension functor in the category of quadratic complexes is, up to isomorphism, the composition of the canonical functor from quadratic to crossed complexes, which sends a 3 -type to its 2-type ([7]), and our suspension functor (Theorem 4.3).

Suspensions in the category of totally free quadratic complexes have a canonical co-H-structure that we study in Section 5. We observe that it is not only a co-H-structure, but a strict cogroup structure, and compute explicitly their structural morphisms in terms of the basis (Theorem [5.6 and Corollary [5.8). These computations are applied in Section 6 to calculate for any $C W$-complex $X$ the natural homomorphism $H_{2} X \rightarrow \wedge^{2} H_{1} X$ induced in the second homology by the map $h: X \rightarrow K\left(H_{1} X, 1\right)$ such that $\pi_{1} h: \pi_{1} X \rightarrow H_{1} X$ is the Hurewicz homomorphism (Theorem 6.1). As a consequence we give abelian presentations for the second quotient $\Gamma_{2} G / \Gamma_{3} G$ of the lower central series of a group $G$ with a given presentation (Proposition [6.4), as well as for the second homology of Leedham-Green ([17]) of $G / \Gamma_{3} G$ in the variety of groups of nilpotency degree 2 (Corollary 6.5).

In the last section we define for any abelian group $A$ a natural element $c_{A} \in$ $\operatorname{Ext}\left(A, S^{2} A\right)$ which vanishes if and only if the Moore space of type $(A, 2)$ has a commutative co-H-structure (Proposition 7.2). The results of Section 5 are applied here to compute an explicit formula of the element $c_{A}$ from a free resolution of $A$ (Theorem 7.5). In this way we show that if $A$ is finitely generated, then $c_{A}$ is trivial if and only if $A$ has an element of order 2 (Corollary 7.6), generalizing a result of Arkowitz and Golasiński ([2]) for cyclic groups.

Further applications of these results to proper homotopy theory will be given in a future paper ([20]). More precisely, the results of Section 6 will be used to show a proper Moore space of degree 2 which does not admit any co-H-structure at all. See [5] and [4] for the construction and basic properties of proper Moore spaces.

\section{Preliminaries}

2.1. Categorical notations. We use the multiplicative notation for the composition of two morphisms $\varphi: X \rightarrow Y, \psi: Y \rightarrow Z$ in a category $\mathbf{C}, \psi \varphi: X \rightarrow Z$. The coproduct of two objects $X, Y$ in $\mathbf{C}$ is denoted by $X \vee Y$, the corresponding inclusions are $X \stackrel{i_{1}}{\hookrightarrow} X \vee Y \stackrel{i_{2}}{\hookrightarrow} Y$. Given two morphisms $X \stackrel{f}{\rightarrow} Z \stackrel{g}{\leftarrow} Y$ in $\mathbf{C}$, $(f, g): X \vee Y \rightarrow Z$ is the unique morphism with $(f, g) i_{1}=f$ and $(f, g) i_{2}=g$. Moreover, $1_{X}$ is the identity morphism in $X$ (we shall omit the subindex if it is 


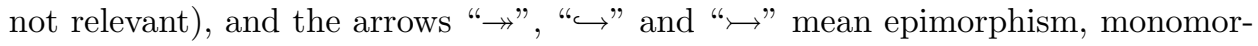
phism and cofibration, respectively. The symbol $\simeq$ will stand for isomorphisms in any category as well as for the homotopy relation between morphisms in homotopy categories, the meaning of this symbol in each case will be clear from the context.

2.2. Quadratic functors. Recall the definition of the following functors in the category $\mathbf{A b}$ of abelian groups

$$
\Gamma, \wedge^{2}, S^{2}: \mathbf{A b} \longrightarrow \mathbf{A b}
$$

The first one is Whitehead's $\Gamma$ functor ([24]), which carries an abelian group $A$ to the abelian group $\Gamma A$ generated by $\{\gamma(a) ; a \in A\}$ with the relations

$$
\begin{gathered}
\gamma(a)=\gamma(-a), \\
\gamma(a+b+c)-\gamma(b+c)-\gamma(a+c)-\gamma(a+b)+\gamma(a)+\gamma(b)+\gamma(c)=0
\end{gathered}
$$

for any $a, b, c \in A$. Let $T: A^{\otimes^{2}} \simeq A^{\otimes^{2}}$ be the involution $T(a \otimes b)=b \otimes a$, the exterior square $\wedge^{2} A$ and symmetric square $S^{2} A$ are defined by the following natural exact sequences

$$
\begin{aligned}
& \Gamma A \stackrel{\tau}{\longrightarrow} A^{\otimes^{2}} \stackrel{q}{\rightarrow} \wedge^{2} A, \\
& A^{\otimes^{2}} \stackrel{1-T}{\longrightarrow} A^{\otimes^{2}} \stackrel{q^{\prime}}{\rightarrow} S^{2} A,
\end{aligned}
$$

where $\tau \gamma(a)=a \otimes a, q(a \otimes b)=a \wedge b$ and $q^{\prime}(a \otimes b)=a b$. There are short splitting exact sequences

$$
\begin{gathered}
A \otimes B \stackrel{l}{\hookrightarrow} \Gamma(A \oplus B) \rightarrow \Gamma A \oplus \Gamma B, \\
A \otimes B \stackrel{\iota}{\hookrightarrow} \wedge^{2}(A \oplus B) \rightarrow \wedge^{2} A \oplus \wedge^{2} B,
\end{gathered}
$$

with $l(a \otimes b)=\gamma\left(i_{1} a+i_{2} b\right)-\gamma\left(i_{1} a\right)-\gamma\left(i_{2} b\right)$ and $\imath(a \otimes b)=i_{1} a \wedge i_{2} b$, that is, $\imath=q\left(i_{1} \otimes i_{2}\right)$. The arrows on the right are induced by the projections of the factors of the direct sum; moreover,

$$
l T=\Gamma\left(i_{2}, i_{1}\right) l
$$

The sequences (2.C) and (2.D) are natural in $A$ and $B$. There is an analogous exact sequence for $S^{2}$ as well, but we shall not use it. Notice that the failure in the additivity of these functors is measured by a biadditive functor, for this reason they are said to be quadratic.

The three functors above are right-exact in the quadratic sense, for $\wedge^{2}$ this property means that given an exact sequence $C \stackrel{f}{\rightarrow} B \stackrel{g}{\rightarrow} A$, then

$$
(C \otimes B) \oplus \wedge^{2} C \stackrel{\left(q(f \otimes 1), \wedge^{2} f\right)}{\longrightarrow} \wedge^{2} B \stackrel{\wedge^{2} g}{\longrightarrow} \wedge^{2} A
$$

is also exact. Furthermore, if $B$ is free abelian with basis $E$ and we choose a total ordering $\preceq$ in $E$, then $\left\{e_{1} \wedge e_{2} ; e_{1} \prec e_{2} \in E\right\}$ is a basis of $\wedge^{2} B$, hence an abelian presentation of $A$ and (2.F) yield an abelian presentation of $\wedge^{2} A$. 
2.3. A review of some group theory. The composition operation in a group $G$ will always be written additively $a+b(a, b \in G)$, even when the group $G$ is not commutative, because we shall use the multiplicative notation for the composition of morphisms in a category and for the generators of the symmetric square; see (2.1) and (2.B). The commutator of two elements in a group $a, b \in G$ is $[a, b]=$ $-a-b+a+b, \Gamma_{n} G$ is the normal subgroup of $G$ generated by commutators of weight $n$. We say that $G$ is of nilpotency degree $\leq n$, or simply a nil $(n)$-group, if $\Gamma_{n+1} G=0$. The abelianization of $G$ is the quotient $G^{a b}=G / \Gamma_{2} G$, and the nilization is $G^{\text {nil }}=G / \Gamma_{3} G$; moreover, we shall denote the natural projections by $p: G \rightarrow G^{a b}$ and $\bar{p}: G \rightarrow G^{n i l}$. These constructions are functorial in the category of groups.

Let gr, nil, ab be the categories of free objects in the varieties of all groups, nil(2)-groups and abelian groups respectively. The object with basis $E$ is denoted in each case $\langle E\rangle,\langle E\rangle^{n i l}$ and $\mathbb{Z}\langle E\rangle$. The "nilization" and "abelianization" defined above restrict to functors

$$
\text { gr } \stackrel{n i l}{\longrightarrow} \text { nil } \stackrel{a b}{\longrightarrow} \text { ab. }
$$

There is a natural central extension

$$
\wedge^{2} \mathbb{Z}\langle E\rangle \stackrel{s}{\hookrightarrow}\langle E\rangle^{n i l} \stackrel{p}{\rightarrow} \mathbb{Z}\langle E\rangle
$$

where $s\left(e_{1} \wedge e_{2}\right)=\left[e_{1}, e_{2}\right]\left(e_{1}, e_{2} \in E\right)$.

More generally, in any nil(2)-group $G$ commutators are central, and the commutator bracket is bilinear, hence there is a natural central homomorphism

$$
w: G^{a b} \otimes G^{a b} \longrightarrow G
$$

defined by $w\left(p(g) \otimes p\left(g^{\prime}\right)\right)=\left[g, g^{\prime}\right]$.

Furthermore, the homomorphism set $\operatorname{Hom}\left(\langle E\rangle^{n i l}, G\right)$ is also a nil(2)-group. The sum of two homomorphisms $\varphi+\psi$ is the unique one such that $(\varphi+\psi)(e)=\varphi(e)+$ $\psi(e)$ for any $e \in E$. In this way morphism sets in nil are nil(2)-groups. The sum and the composition of morphisms in nil satisfy the following rules (see 10]):

(i) $\psi\left(\varphi_{1}+\varphi_{2}\right)=\psi \varphi_{1}+\psi \varphi_{2}$,

(ii) the symbol $\left(\psi_{1} \mid \psi_{2}\right)_{\varphi}=\left(\psi_{1}+\psi_{2}\right) \varphi-\psi_{2} \varphi-\psi_{1} \varphi$ is linear in $\psi_{1}$ and $\psi_{2}$; moreover, it only depends on $\psi_{1}^{a b}$ and $\psi_{2}^{a b}$ and lies in the commutator subgroup of the corresponding homomorphism group,

(iii) $(-\psi) \varphi=-\psi \varphi+(\psi \mid \psi)_{\varphi}$.

By using (ii) and (iii) one can check that

(iv) if $\psi_{1}^{a b}=\psi_{2}^{a b}$, then $\left(\psi_{1}-\psi_{2}\right) \varphi=\psi_{1} \varphi-\psi_{2} \varphi$.

Given any two groups $\pi, G$ we say that $G$ is a $\pi$-group if $\pi$ acts (on the right) on $G$ by automorphisms, the action is written exponentially $a^{\alpha}(a \in G, \alpha \in \pi)$. Notice that $G$ is canonically a $G$ group acting on itself by conjugation $a^{b}=-b+a+b$ $(a, b \in G)$. If $H$ is a $\pi^{\prime}$-group and $v: \pi \rightarrow \pi^{\prime}, \psi: G \rightarrow H$ are homomorphisms, we say that $\psi$ is $v$-equivariant if $\psi\left(a^{\alpha}\right)=\psi(a)^{v(\alpha)}$ for any $a \in G, \alpha \in \pi$. When $\pi=\pi^{\prime}$ and $v=1$ we simply say that $\psi$ is $\pi$-equivariant.

If we have two groups $G, H$ acting one on each other, their non-abelian tensor product $G \otimes H$ is the group generated by the symbols $g \otimes h$ with relations

$$
\left(g+g^{\prime}\right) \otimes h=g^{g^{\prime}} \otimes h^{g^{\prime}}+g^{\prime} \otimes h, g \otimes\left(h+h^{\prime}\right)=g \otimes h^{\prime}+g^{h^{\prime}} \otimes h^{h^{\prime}}
$$

for any $g, g^{\prime} \in G$ and $h, h^{\prime} \in H$. Here the relations are slightly different from those in 15 since we consider right-actions. This translation will be needed whenever 
we use this construction and related results in [15]. If the actions are both trivial, then $G \otimes H=G^{a b} \otimes H^{a b}$ is the usual tensor product of the abelianized groups. Moreover, one can easily check that this tensor product is functorial with respect to homomorphisms $\varphi: G \rightarrow G^{\prime}, \psi: H \rightarrow H^{\prime}$ such that $\varphi$ is $\psi$-equivariant and $\psi$ is $\varphi$-equivariant, $\varphi \otimes \psi: G \otimes H \rightarrow G^{\prime} \otimes H^{\prime}: g \otimes h \mapsto \varphi(g) \otimes \psi(h)$. Furthermore, there is a canonical homomorphism induced by the commutator bracket

$$
w^{\prime}: G \otimes G \longrightarrow G: g \otimes g^{\prime} \mapsto\left[g, g^{\prime}\right] .
$$

\section{A SUSPENSION FUNCTOR FROM CROSSED MODULES TO REDUCED QUADRATIC MODULES}

In this section we are going to construct a functor from the category of crossed modules to the category of reduced quadratic modules which we shall call suspension functor. We shall justify the name "suspension" in the next section. We also give here a brief account of the definitions and basic properties of these algebraic objects; see [7] for more details.

Recall that a precrossed module $\partial$ is an $N$-group $M$ together with an $N$-equivariant homomorphism $\partial: M \rightarrow N$. A morphism of precrossed modules $(\psi, v): \partial \rightarrow \partial^{\prime}$ is a commutative square of homomorphisms

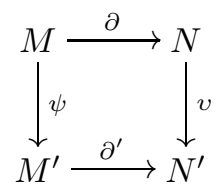

such that $\psi$ is $v$-equivariant. The Peiffer commutator of two elements $x, y \in M$ is $\llbracket x, y \rrbracket=-x-y+x+y^{\partial x}$, and $\mathrm{P}_{n} \partial \subset M$ is the subgroup generated by Peiffer commutators of weight $n$. We can define the nilpotency degree of a precrossed module just as in the case of groups by using here $\mathrm{P}_{n} \partial$ instead of $\Gamma_{n} G$; see [9]. We call nil $(n)$-modules to precrossed modules of nilpotency degree $\leq n$, nil(1)modules are usually termed crossed modules. The crossed module $\partial^{\text {cr }}$ associated to a precrossed module $\partial$ is the quotient

$$
\partial^{c r}: M^{c r}=M / \mathrm{P}_{2} \partial \rightarrow N,
$$

and the related nil(2)-module is

$$
\partial^{n i l}: M / P_{3} \partial \rightarrow N
$$

Remark 3.1. Notice that a precrossed module $\partial$ with $N=0$ is just a group $M$, in this case $\mathrm{P}_{n} \partial=\Gamma_{n} M$. In general, $\Gamma_{n} \operatorname{Ker} \partial \subset \mathrm{P}_{n} \partial$, hence if $\partial$ is a nil $(n)$-module Ker $\partial$ is a $\operatorname{nil}(n)$-group.

If $\partial: M \rightarrow N$ is a crossed module and we consider $M$ acting on $N$ via $\partial$ and conjugation, then one easily checks that the actions are compatible in the sense of [15], and hence the following homomorphism

$$
M \otimes N \stackrel{\varpi}{\longrightarrow} M: m \otimes n \mapsto-m+m^{n}
$$

is well defined and natural, and verifies

- $\varpi(1 \otimes \partial)=w^{\prime}: M \otimes M \rightarrow M$,

- $\partial \varpi=w^{\prime}(\partial \otimes 1): M \otimes N \rightarrow N$. 
In [7] a quadratic module $(\omega, \delta, \partial)$ is defined as a diagram of $N$-groups

$$
C \otimes C \stackrel{\omega}{\rightarrow} L \stackrel{\delta}{\rightarrow} M \stackrel{\partial}{\rightarrow} N
$$

where $\partial$ is a nil(2)-module, $\phi: M \rightarrow\left(M^{c r}\right)^{a b}=C$ is the natural projection (which induces a unique action of $N$ on $C$ such that $\phi$ is $N$-equivariant), $C \otimes C$ has the diagonal action, and the following equalities hold $(a, b \in L ; x, y \in M)$ :

(i) $\partial \delta=0$,

(ii) $\llbracket x, y \rrbracket=\delta \omega(\phi(x) \otimes \phi(y))$,

(iii) $a^{\partial x}=a+\omega(1+T)(\phi \delta(a) \otimes \phi(x))$,

(iv) $[a, b]=\omega(\phi \delta(a) \otimes \phi \delta(b))$.

Remark 3.2. Notice that $\omega$ is central and $L$ is a nil(2)-group by (iv).

A morphism of quadratic modules $(\zeta, \psi, v):(\omega, \delta, \partial) \rightarrow\left(\omega^{\prime}, \delta^{\prime}, \partial^{\prime}\right)$ is a commutative diagram

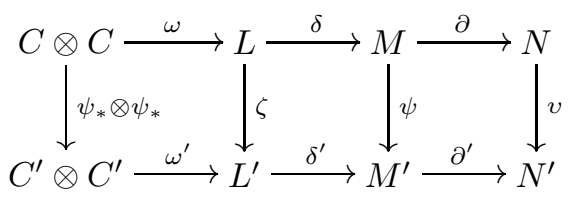

of $v$-equivariant homomorphisms with $\psi_{*}: C \rightarrow C^{\prime}$ induced by $\psi$.

We are mainly interested in reduced quadratic modules, which are those with $N=0$. In this case $M$ is also a nil(2)-group, $C=M^{a b}$ and conditions above reduce to the following:

(i) $[x, y]=\delta \omega(\phi(x) \otimes \phi(y))$,

(ii) $\omega(1+T)(\phi \delta(a) \otimes \phi(x))=0$,

(iii) $[a, b]=\omega(\phi \delta(a) \otimes \phi \delta(b))$.

We shall also use the central push-out $P$ of a diagram of groups $H \stackrel{g}{\leftarrow} G \stackrel{f}{\rightarrow} A$ with $A$ abelian. Namely, $P$ is a group which fits into a commutative square

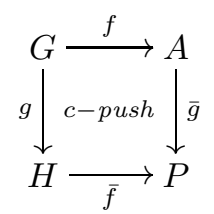

with $\bar{g}$ central, and satisfies the following universal property: for any pair of homomorphisms $\varphi: H \rightarrow K, \psi: A \rightarrow K$ with $\psi$ central and $\varphi g=\psi f$ there exists a unique homomorphism $\phi: P \rightarrow K$ with $\varphi=\phi \bar{f}$ and $\psi=\phi \bar{g}$. The group $P$ can be constructed as the quotient of $H \times A$ by the normal subgroup generated by the elements $(g(x),-f(x)), x \in G$.

Proposition 3.3. There exists a suspension functor $\tilde{\Sigma}$ from crossed to reduced quadratic modules which sends $\partial: M \rightarrow N$ to the reduced quadratic module $\tilde{\Sigma} \partial=$ 
$(\omega, \delta)$ given by the following commutative diagram:

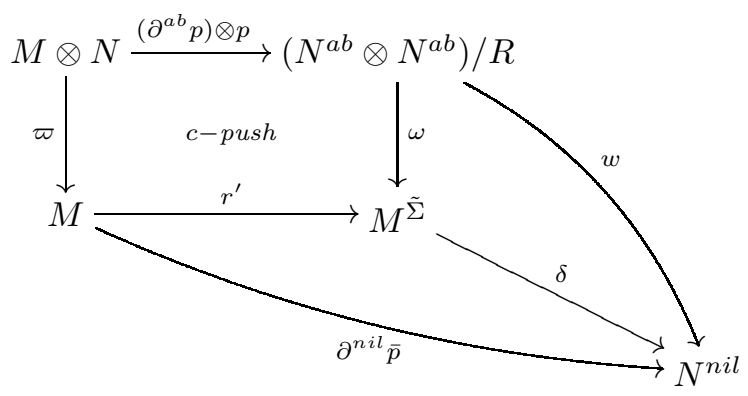

Here $R$ is the image of $(1+T)\left(\partial^{a b} \otimes 1\right): M^{a b} \otimes N^{a b} \rightarrow N^{a b} \otimes N^{a b}$.

Proof. The natural homomorphism $w$ defined in (2.H) factors through $R$, since commutators are anticommutative. Moreover, $\delta$ exists and $(\omega, \delta)$ is a reduced quadratic module by the properties of $\varpi$ in (3.A) and central push-outs. Furthermore, the diagram above is clearly natural in the category of crossed modules, hence $\tilde{\Sigma}$ is a functor and the proof is finished.

We say that a precrossed module $\partial: M \rightarrow N$ is totally free with basis $\varphi:\langle E\rangle \rightarrow$ $\langle F\rangle$ if $N=\langle F\rangle$ and a homomorphism $r:\langle E\rangle \rightarrow M$ is given such that if $\partial^{\prime}$ is another precrossed module, then any commutative diagram of groups

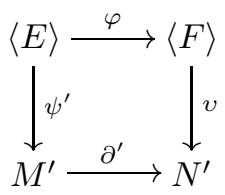

extends to a unique morphism $(\psi, v): \partial \rightarrow \partial^{\prime}$ of precrossed modules with $\psi r=\psi^{\prime}$. This object is constructed in the following way: $M=\operatorname{Ker}[(0,1):\langle E\rangle * N \rightarrow N]$ with $N$ acting on $M$ by conjugation in the free product $\langle E\rangle * N$, and

$$
\partial: M \subset\langle E\rangle * N \stackrel{(\varphi, 1)}{\longrightarrow} N .
$$

The totally free crossed module (resp. nil(2)-module) with basis $\varphi$ is $\partial^{c r}$ (resp. $\left.\partial^{n i l}\right)$.

Similarly, a quadratic module $(\omega, \delta, \partial)$ is totally free with basis $\langle D\rangle \rightarrow M$ if the nil(2)-module $\partial$ is totally free and there is a homomorphism $r:\langle D\rangle \rightarrow L$ satisfying the universal property analogue to the case of precrossed modules.

A reduced quadratic module $M^{a b} \otimes M^{a b} \stackrel{\omega}{\rightarrow} L \stackrel{\delta}{\rightarrow} M$ is totally free with basis $\varphi:\langle E\rangle^{n i l} \rightarrow\langle F\rangle^{n i l}$ if $M=\langle F\rangle^{n i l}$ and a homomorphism $r:\langle E\rangle^{n i l} \rightarrow L$ is given satisfying the obvious universal property. This object is constructed by the following 
diagram:

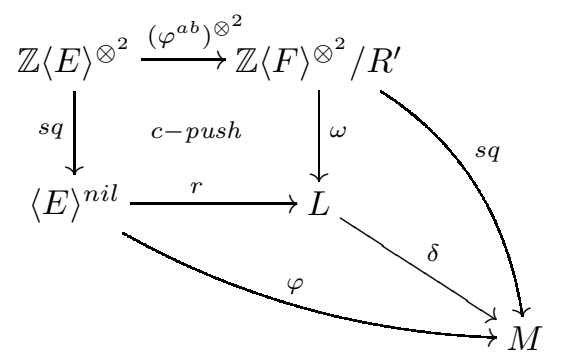

Here $R^{\prime}$ is the image of $(1+T)\left(\varphi^{a b} \otimes 1\right): \mathbb{Z}\langle E\rangle \otimes \mathbb{Z}\langle F\rangle \rightarrow \mathbb{Z}\langle F\rangle^{\otimes^{2}}$.

The next proposition shows that the suspension functor defined in (3.3) preserves totally free objects.

Proposition 3.4. If the crossed module $\partial: M \rightarrow N$ is totally free with basis $\varphi:\langle E\rangle \rightarrow\langle F\rangle$, then the reduced quadratic module $\tilde{\Sigma} \partial=(\omega, \delta)$ is totally free with basis $\varphi^{\text {nil }}$.

Proof. Write $\bar{\partial}: \bar{M} \rightarrow N$ for the totally free precrossed module with basis $\varphi$, then $\bar{M}=\underset{f \in\langle F\rangle}{*}\left\langle E^{f}\right\rangle \subset\langle E\rangle *\langle F\rangle$ and $M^{\tilde{\Sigma}}$ in Proposition 3.3 is the quotient of $\bar{M} \times$ $\left(\mathbb{Z}\langle F\rangle^{\otimes^{2}} / R\right)$ by the normal subgroup generated by the elements

$$
\left(-e^{\prime}-e+e^{\prime}+e^{\varphi e^{\prime}}, 0\right),\left(-e+e^{f},-\varphi^{a b} p(e) \otimes p(f)\right)
$$

for any $e, e^{\prime} \in\langle E\rangle, f \in\langle F\rangle$. Hence in the quotient we have the equality $\left(e^{f}, 0\right)=$ $\left(e, \varphi^{a b} p(e) \otimes p(f)\right)$, and therefore $M^{\tilde{\Sigma}}$ is also the quotient of $\langle E\rangle \times\left(\mathbb{Z}\langle F\rangle^{\otimes^{2}} / R\right)$ by the elements $\left(\left[e^{\prime}, e\right], \varphi^{a b} p(e) \otimes \varphi^{a b} p\left(e^{\prime}\right)\right)$ for any $e, e^{\prime} \in\langle E\rangle$. In particular, $\left(\left[e,\left[e^{\prime}, e^{\prime \prime}\right]\right], 0\right)$ is a relation for every $e, e^{\prime}, e^{\prime \prime} \in\langle E\rangle$, then $M^{\tilde{\Sigma}}$ is the quotient of $\langle E\rangle^{n i l} \times\left(\mathbb{Z}\langle F\rangle^{\otimes^{2}} / R\right)$ by the relations $\left(-\left[e, e^{\prime}\right], \varphi^{a b} p(e) \otimes \varphi^{a b} p\left(e^{\prime}\right)\right)$ for any $e, e^{\prime} \in\langle E\rangle^{n i l}$, since $\left[e^{\prime}, e\right]=$ $-\left[e, e^{\prime}\right]$. Moreover, by an easy computation one can check that $R=R^{\prime}$, hence $M^{\tilde{\Sigma}}$ coincides with $L$ in the central push-out diagram (3.C). The proof is now complete.

\section{Crossed and quadratic complexes. Suspensions}

Crossed (resp. quadratic) complexes are special chain complexes of groups with a crossed (resp. quadratic) module structure in low dimensions (see [7]). These algebraic objects are used to encode homotopical information of spaces, such as the 2 -type and the 3-type. In this section we extend to those complexes the suspension functor defined in the previous section, and give a topological interpretation of this algebraic functor.

A crossed complex is a positive chain complex of groups $\rho=\left(\rho_{*}, d_{*}\right)$ such that $d_{2}: \rho_{2} \rightarrow \rho_{1}$ is a crossed module, $\rho_{n}(n>2)$ is a (right) module over Coker $d_{2}$ (and hence over $\rho_{1}$ through the natural projection $\rho_{1} \rightarrow$ Coker $d_{2}$ ), and the differential $d_{*}$ is $\rho_{1}$-equivariant. Similarly, a quadratic complex $\sigma=\left(\sigma_{*}, d_{*}, \omega\right)$ is a positive chain complex of groups $\left(\sigma_{*}, d_{*}\right)$ and a homomorphism $\omega: C_{2} \otimes C_{2} \rightarrow \sigma_{3}$ such that $\left(\omega, d_{3}, d_{2}\right)$ is a quadratic module, $\sigma_{n}(n>3)$ is a module over Coker $d_{2}$, and the differential $d_{*}$ is $\sigma_{1}$-equivariant. The homotopy groups of $\rho$ and $\sigma$ are

$$
\pi_{n} \rho=H_{n}\left(\rho_{*}, d_{*}\right), \pi_{n} \sigma=H_{n}\left(\sigma_{*}, d_{*}\right) .
$$


A morphism of crossed complexes is a morphism of chain complexes

$$
f=\left\{f_{n}\right\}_{n \geq 1}: \rho \rightarrow \rho^{\prime}
$$

such that $f_{n}$ is $f_{1}$-equivariant $(n \geq 1)$, and morphisms of quadratic complexes $f=$ $\left\{f_{n}\right\}_{n \geq 1}: \sigma \rightarrow \sigma^{\prime}$ are morphisms of chain complexes with $\left(f_{3}, f_{2}, f_{1}\right):\left(\omega, d_{3}, d_{2}\right) \rightarrow$ $\left(\omega^{\prime}, d_{3}^{\prime}, d_{2}^{\prime}\right)$ a morphism of quadratic modules and $f_{n}$ an $f_{1}$-equivariant homomorphism $(n \geq 1)$. A morphism of crossed or quadratic complexes is said to be a weak equivalence if it induces isomorphisms in homotopy groups.

We say that a crossed or quadratic complex is reduced if the 1-dimensional group is trivial. Notice that reduced crossed complexes are simply chain complexes of abelian groups, while reduced quadratic complexes have a reduced quadratic module in low dimensions with a chain complex of abelian groups attached in dimensions $>3$.

In the next proposition we extend the suspension functor defined in Proposition 3.3 to a functor between complexes.

Proposition 4.1. There is a suspension functor $\tilde{\Sigma}$ from crossed to reduced quadratic complexes which sends $\rho=\left(\rho_{*}, d_{*}\right)$ to the reduced quadratic complex $\tilde{\Sigma} \rho=$ $\left((\tilde{\Sigma} \rho)_{*}, d_{*}^{\tilde{\Sigma}}, \omega\right)$ with $\left(\omega, d_{3}^{\tilde{\Sigma}}\right)=\tilde{\Sigma} d_{2},(\tilde{\Sigma} \rho)_{n+1}=\rho_{n} \otimes_{\pi_{1} \rho} \mathbb{Z}$ for $n>2$, where $\mathbb{Z}$ is the trivial $\pi_{1} \rho$-module, and $d_{n+1}^{\tilde{\Sigma}}=d_{n} \otimes_{\pi_{1} \rho} \mathbb{Z}$ for $n>3$. Moreover, the homomorphism $d_{4}^{\tilde{\Sigma}}$ is the unique one which extends commutatively the following diagram:

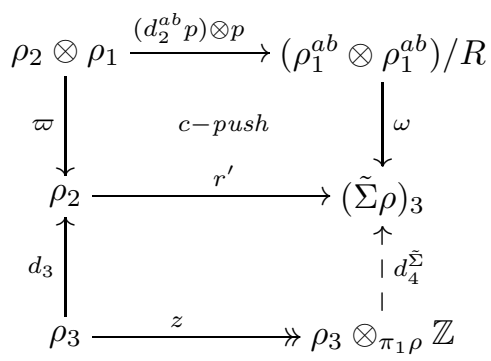

where $z$ is the natural projection $z(x)=x \otimes 1\left(x \in \rho_{3}\right)$.

Proof. The filler $d_{4}^{\tilde{\Sigma}}$ exists and is unique provided $d_{3}(\operatorname{Ker} z) \subset \operatorname{Ker} r^{\prime}$. This inclusion is satisfied, because Ker $z$ is generated by the elements $-x+x^{\alpha}\left(x \in \rho_{3}, \alpha \in \rho_{1}\right)$, and

$$
\begin{aligned}
r^{\prime} d_{3}\left(-x+x^{\alpha}\right) & =r^{\prime}\left(-d_{3}(x)+d_{3}(x)^{\alpha}\right) \\
& =r^{\prime} \varpi\left(d_{3}(x) \otimes \alpha\right) \\
& =\omega\left(d_{2}^{a b} p d_{3}(x) \otimes p \alpha\right) \\
& =\omega\left(p d_{2} d_{3}(x) \otimes p \alpha\right) \\
& =0 .
\end{aligned}
$$

It is readily checked that $d^{\tilde{\Sigma}} d^{\tilde{\Sigma}}=0$, since $d d=0$, hence $\tilde{\Sigma} \rho$ is a reduced quadratic complex. Furthermore, $\tilde{\Sigma}$ is a functor because the diagram of the statement is natural in the category of crossed complexes.

A crossed (resp. quadratic) complex $\rho$ (resp. $\sigma$ ) is said to be totally free if $d_{2}$ (resp. $\left.\left(\omega, d_{3}, d_{2}\right)\right)$ is a totally free crossed (resp. quadratic) module and $\rho_{n}$ (resp. $\left.\sigma_{n}\right)$ is a free module over Coker $d_{2}$ for $n>2$ (resp. $\left.n>3\right)$. 
We say that $\left\{E_{n}\right\}_{n \geq 1}$ is the basis of a totally free quadratic complex $\sigma=$ $\left(\sigma_{*}, d_{*}, \omega\right)$ if $E_{n} \subset \sigma_{n}$ is a subset such that

(i) $\sigma_{1}=\left\langle E_{1}\right\rangle$,

(ii) $d_{2}$ induces a group homomorphism $\left\langle E_{2}\right\rangle \rightarrow\left\langle E_{1}\right\rangle$ which is the basis of the nil(2)-module $d_{2}$,

(iii) the homomorphism $\left\langle E_{3}\right\rangle \rightarrow \sigma_{2}$ induced by $d_{3}$ is the basis of the quadratic module $\left(\omega, d_{3}, d_{2}\right)$,

(iv) $E_{n}(n \geq 4)$ is the basis of the free $\pi_{1} \sigma$-module $\sigma_{n}$.

If $\sigma$ is reduced, then $E_{1}=\emptyset$ is the empty set and conditions above reduce to the following:

(i) $\sigma_{2}=\left\langle E_{2}\right\rangle^{n i l}$,

(ii) $d_{3}$ induces a homomorphism $\left\langle E_{3}\right\rangle^{\text {nil }} \rightarrow\left\langle E_{2}\right\rangle^{\text {nil }}$ which is the basis of the reduced quadratic module $\left(\omega, d_{3}\right)$,

(iii) $E_{n}(n \geq 4)$ is the basis of the free abelian group $\sigma_{n}$.

Remark 4.2. The basis of crossed complexes is defined in a similar way, and has analogous properties. Moreover, a totally free crossed or quadratic complex is completely determined by the basis sets and the values of the differential over the basis. Furthermore, if $f: \sigma \rightarrow \sigma^{\prime}$ is a morphism of quadratic complexes and $\sigma$ is totally free, $f$ is also determined by its values on the basis. The same happens in the crossed case.

The categories $\mathbf{H}, \mathbf{Q}$ of totally free crossed and quadratic complexes, respectively, are homotopy categories; in fact, $I$-categories with the structure given in [7. The zero object in both categories is that with the trivial group in each dimension, and the basis of the coproduct of two objects is the coproduct (disjoint union) of the basis. Moreover, the inclusion of a factor into a coproduct is induced by the inclusion of its basis; see also the construction of homotopy push-outs in these categories given in 7], III.4.17 and IV.5.5.

The basis of the cylinder $I \sigma$ of a quadratic complex $\sigma$ with basis $\left\{E_{n}\right\}_{n \geq 1}$ is given by the sets $\left\{E_{n}^{\prime}\right\}_{n \geq 1}$ with

$$
\begin{gathered}
E_{n}^{\prime}=i_{0} E_{n} \vee s E_{n-1} \vee i_{1} E_{n} \quad(n \geq 2), \\
E_{1}^{\prime}=i_{0} E_{1} \vee i_{1} E_{1} .
\end{gathered}
$$

Here $i_{t} E_{n}, s E_{n}$ are copies of $E_{n}(n \geq 1, t=0,1)$ and $\vee$ is the disjoint union of sets. The natural top and bottom inclusions of $\sigma$ into the cylinder $i_{t}: \sigma \longmapsto I \sigma$ $(t=0,1)$ are induced by the basis inclusions $E_{n}=i_{t} E_{n} \subset E_{n}^{\prime}$, and the natural projection $p: I \sigma \rightarrow \sigma$ by $p i_{t}=1$ and $p_{n+1}(s x)=0\left(t=0,1 ; x \in E_{n}, n \geq 1\right)$. The differential in the cylinder is defined by the differential of $\sigma$ and the following equalities $\left(x \in E_{n}, n \geq 1, t=1,2\right)$ :

$$
d_{n} i_{t}(x)=i_{t} d_{n}(x), d_{n+1}(s x)=-i_{0}(x)+i_{1}(x)-S_{n-1} d_{n}(x) .
$$

Here $S_{0} d_{1}(x)=0$ and $S_{n}: \sigma_{n} \rightarrow(I \sigma)_{n+1}(n \geq 1)$ is the function defined by $S_{n} x=s x\left(x \in E_{n}\right)$ and the fact that it is an $i_{0}$-equivariant homomorphism for $n \geq 3$, an $i_{0}$-crossed homomorphism for $n=1$, and an $\left(S_{1}, i_{0}, i_{1}\right)$-quadratic operator for $n=2$.

Recall that, if $G$ is a $\pi^{\prime}$-group and $\varphi: \pi \rightarrow \pi^{\prime}$ is a homomorphism, $\psi: \pi \rightarrow G$ is a $\varphi$-crossed homomorphism if $\psi(a+b)=\psi(a)^{\varphi b}+\psi(b)$ for any $a, b \in \pi$. The definition of quadratic operator is more complicated, we refer to 7], IV.4.3. 
Given a morphism $f: \sigma \rightarrow \sigma^{\prime}$ in $\mathbf{Q}$, the induced morphism $I f: I \sigma \rightarrow I \sigma^{\prime}$ is the unique one with $(I f) i_{t}=i_{t} f(t=0,1)$ and $(I f)_{n+1}(s x)=S_{n} f_{n}(x)\left(x \in E_{n}, n \geq 1\right)$.

The suspension functor in $\mathbf{Q}$, as in any $I$-category, is defined by $\Sigma \sigma=I \sigma / \sigma \vee \sigma$, the cofiber of the natural morphism $\left(i_{0}, i_{1}\right): \sigma \vee \sigma \longmapsto I \sigma$. In order to relate this suspension functor with that defined in Proposition 4.1 recall that there is a functor $\lambda$ from quadratic complexes to crossed complexes which sends $\sigma=\left(\sigma_{*}, d_{*}, \omega\right)$ to the crossed complex $\lambda \sigma=\left((\lambda \sigma)_{*}, d_{*}^{\lambda}\right)$ such that $\left((\lambda \sigma)_{*}, d_{*}^{\lambda}\right)$ coincides with $\left(\sigma_{*}, d_{*}\right)$ in dimensions $>3$,

$$
\begin{gathered}
d_{4}^{\lambda}:(\lambda \sigma)_{4}=\sigma_{4} \stackrel{d_{4}}{\rightarrow} \sigma_{3} \rightarrow \text { Coker } \omega=(\lambda \sigma)_{3}, \\
d_{2}^{\lambda}=d_{2}^{c r}:(\lambda \sigma)_{2}=\sigma_{2}^{c r} \rightarrow \sigma_{1}=(\lambda \sigma)_{1},
\end{gathered}
$$

and $d_{3}^{\lambda}$ fits into the following commutative square:

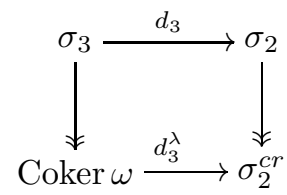

(see IV.3.2 in [7). This functor preserves totally free complexes; moreover, if $\left\{E_{n}\right\}_{n \geq 1}$ is the basis of $\sigma$, then it is also the basis of $\lambda \sigma$, and $\lambda I \sigma=I \lambda \sigma$.

Suspensions in $\mathbf{H}$ are just chain complexes of free abelian groups which are trivial in dimensions $\leq 1$, so they are not of much interest. The main result of this section is the following.

Theorem 4.3. There is a natural equivalence between the functors

$$
\mathbf{Q} \stackrel{\Sigma}{\longrightarrow} \mathbf{Q} \text { and } \mathbf{Q} \stackrel{\lambda}{\longrightarrow} \mathbf{H} \stackrel{\tilde{\Sigma}}{\longrightarrow} \mathbf{Q} .
$$

In order to prove this theorem we first state several lemmas.

The following lemma follows directly from Proposition 3.4 and the construction of $\tilde{\Sigma}$ in Proposition 4.1.

Lemma 4.4. If $\rho$ is a totally free crossed module with basis $\left\{E_{n}\right\}_{n \geq 1}$, then the reduced quadratic module $\tilde{\Sigma} \rho$ is totally free with basis $\left\{E_{n}^{\tilde{\Sigma}}\right\}_{n \geq 1}, E_{1}^{\tilde{\Sigma}}=\emptyset, E_{n}^{\tilde{\Sigma}}=$ $E_{n-1}(n \geq 2)$.

By observing the explicit constructions of the cylinder functor, the suspension functor $\Sigma$, and homotopy push-outs in $\mathbf{Q}(7])$ one easily checks that

Lemma 4.5. If $\left\{E_{n}\right\}_{n>1}$ is the basis of the quadratic complex $\sigma$, then the basis of $\Sigma \sigma$ is $\left\{E_{n}^{\Sigma}\right\}_{n \geq 1}$ with $E_{1}^{\Sigma}=\emptyset$ and $E_{n}^{\Sigma}=E_{n-1}$ for $n \geq 2$. Moreover, if $\varrho: I \sigma \rightarrow$ $I \sigma / \sigma \vee \sigma=\Sigma \sigma$ is the natural quotient morphism, then the restriction of $\varrho_{n}$ induces the identity $\varrho_{n}: s E_{n-1}=E_{n-1}=E_{n}^{\Sigma}(n \geq 2)$.

In the next lemma we determine the low-dimensional reduced quadratic module of a suspended quadratic complex.

Lemma 4.6. If $\bar{\varphi}:\left\langle E_{2}\right\rangle \rightarrow\left\langle E_{1}\right\rangle$ is the basis of the nil(2)-module $d_{2}: \sigma_{2} \rightarrow \sigma_{1}$ and $\varphi=\bar{\varphi}^{\text {nil }}$, then $-\varphi:\left\langle E_{2}\right\rangle^{n i l} \rightarrow\left\langle E_{1}\right\rangle^{\text {nil }}$ is the basis of the low-dimensional reduced quadratic module of $\Sigma \sigma$,

$$
\mathbb{Z}\left\langle E_{1}\right\rangle^{\otimes^{2}} \stackrel{\omega^{\Sigma}}{\longrightarrow}(\Sigma \sigma)_{3} \stackrel{d_{3}^{\Sigma}}{\longrightarrow}(\Sigma \sigma)_{2}=\left\langle E_{1}\right\rangle^{n i l} .
$$


Proof. By (4.5) we only need to prove that the composition

$$
E_{2}=s E_{2} \subset(I \sigma)_{3} \stackrel{d_{3}}{\rightarrow}(I \sigma)_{2} \stackrel{\varrho_{2}}{\rightarrow}(\Sigma \sigma)_{2}=\left\langle E_{1}\right\rangle^{n i l}
$$

sends $x \in E_{2}$ to $-\varphi(x) \in\left\langle E_{1}\right\rangle^{n i l}$. By construction of the differential of $I \sigma$ and the suspension functor we have that

$$
\varrho_{2} d_{3}(s x)=-\varrho_{2} S_{1} \bar{\varphi}(x) .
$$

Moreover, since $\varrho i_{0}=0, \varrho_{2} S_{1}$ is in fact a group homomorphism, and given $y \in E_{1}$, $\varrho_{2} S_{1}(y)=y$, so $\varrho_{2} S_{1}=\bar{p}:\left\langle E_{1}\right\rangle \rightarrow\left\langle E_{1}\right\rangle^{n i l}$ is the natural projection and $\varrho_{2} d_{3}(s x)=$ $-\varrho_{2} S_{1} \bar{\varphi}(x)=-\bar{p} \bar{\varphi}(x)=-\varphi(x)$.

Now we are ready to prove Theorem 4.3 .

Proof of Theorem 4.3. Let $\sigma=\left(\sigma_{*}, d_{*}, \omega\right)$ be a quadratic complex. We claim that $(\Sigma \sigma)_{n}=(\tilde{\Sigma} \lambda \sigma)_{n}$ and the natural equivalence is given by the morphism $\chi: \Sigma \sigma \rightarrow$ $\tilde{\Sigma} \lambda \sigma$ with $\chi_{n}=(-1)^{n}$ for $n \neq 3, \chi_{3} \omega=\omega$ and $\chi_{3} r=r(-1)$.

Given $\varphi \in$ nil, the reduced quadratic modules with basis $-\varphi$ and $\varphi,\left(\omega_{1}, \delta_{1}\right)$ and $\left(\omega_{2}, \delta_{2}\right)$ respectively, are defined by the same groups, $\omega_{1}=\omega_{2}$ but $\delta_{1} \neq \delta_{2}$ in general, here we use (3.C) and the fact that $\left(-\varphi^{a b}\right)^{\otimes^{2}}=\left(\varphi^{a b}\right)^{\otimes^{2}}$. Moreover, $\left(\chi_{3}, 1,0\right):\left(\omega_{1}, \delta_{1}\right) \rightarrow\left(\omega_{2}, \delta_{2}\right)$ is an isomorphism of quadratic modules. Therefore, the equalities $(\Sigma \sigma)_{n}=(\tilde{\Sigma} \lambda \sigma)_{n}$ and $\chi_{2} d_{3}^{\Sigma}=d_{3}^{\tilde{\Sigma} \lambda} \chi_{3}$ follow from Proposition 3.4 and Lemmas $4.4,4.5$ and 4.6 .

Since $\varrho i_{t}=0(t=0,1)$, for $n \geq 5$ we have that $\varrho_{n} S_{n-1}: \sigma_{n-1} \rightarrow(\Sigma \sigma)_{n}=$ $(\tilde{\Sigma} \lambda \sigma)_{n}=\sigma_{n-1} \otimes_{\pi_{1} \sigma} \mathbb{Z}$ is the natural projection $x \mapsto x \otimes 1$ and $d_{n+1}^{\Sigma}(x)=$ $-\varrho_{n} S_{n-1} d_{n}(x)$, so $d_{n+1}^{\Sigma}=-d_{n} \otimes_{\pi_{1} \sigma} \mathbb{Z}=-d_{n+1}^{\tilde{\Sigma} \lambda}$ and $\chi_{n} d_{n+1}^{\Sigma}=d_{n+1}^{\tilde{\Sigma} \lambda} \chi_{n+1}$. Furthermore, $\varrho_{4} S_{3}: \sigma_{3} \rightarrow(\Sigma \sigma)_{4}=(\tilde{\Sigma} \lambda \sigma)_{4}=($ Coker $\omega) \otimes_{\pi_{1} \sigma} \mathbb{Z}$ is also the projection homomorphism onto the quotient, and if $x \in E_{4}$, then $d_{5}^{\Sigma}(x)=-\varrho_{4} S_{3} 4 d_{4}(x)=$ $-d_{5}^{\tilde{\Sigma} \lambda}(x)$, hence $\chi_{4} d_{5}^{\Sigma}=d_{5}^{\tilde{\Sigma} \lambda} \chi_{5}$. The map $S_{2}$ is an $\left(S_{1}, i_{0}, i_{1}\right)$-quadratic operator in the sense of [7, IV.4.3 (see [7], IV.4.10 (5)); in addition $\varrho i_{0}=0=\varrho i_{1}$ and $\varrho_{2} S_{1}=\bar{p}$ (for this last equality see the proof of Lemma 4.6). Then $\varrho_{3} S_{2}$ is a $(\bar{p}, 0,0)$-quadratic operator. Let $\xi$ be the composite $\sigma_{2} \rightarrow \sigma_{2}^{c r}=(\lambda \sigma)_{2} \stackrel{r^{\prime}}{\rightarrow}(\widetilde{\Sigma} \lambda \sigma)_{3}$; see Proposition 3.3. By using property (iii) in the definition of a reduced quadratic module (see Section 3) and Proposition 3.3 it is easy to see that the map $-\chi_{3} \xi: \sigma_{2} \rightarrow(\widetilde{\Sigma} \lambda \sigma)_{3}=\left(\Sigma \sigma_{3}\right): x \mapsto-\chi_{3} \xi(x)$ is also a $(\bar{p}, 0,0)$-quadratic operator. Moreover, given $e \in E_{2}, \varrho_{3} S_{2}(e)=e=-\chi_{3} \xi(e)$ and therefore by [7, IV.4.5, $\varrho_{2} S_{1}=-\chi_{3} \xi$. In particular by Proposition 4.1 we have that for $x \in E_{3}, d_{4}^{\tilde{\Sigma} \lambda} \chi_{4}(x)=$ $d_{4}^{\widetilde{\Sigma} \lambda} x=\xi d_{3}(x)=\chi_{3} \chi_{3} \xi d_{3}(x)=\chi_{3}\left(-\varrho S_{2} d_{3}(x)\right)=\chi_{3} d_{4}^{\Sigma}(x)$. Here we use the obvious fact that $\chi_{3} \chi_{3}=1$. The proof is now finished.

Let $\mathbf{C W}$ be the category of CW-complexes with trivial 0-skeleton and cellular maps. From the homotopy point of view this category is equivalent to the category of all connected spaces, therefore in this paper "space" will mean CW-complex as above, and maps between them will always be cellular.

Given a space $X$ there are defined objects $\rho(X), \sigma(X)$ in $\mathbf{H}$ and $\mathbf{Q}$, respectively, such that $\rho(X)=\lambda \sigma(X)$; see [7. Moreover, the basis of these objects is the set of cells of $X$. In fact, they are functors $\rho: \mathbf{C W} \rightarrow \mathbf{H}$ and $\sigma: \mathbf{C W} \rightarrow \mathbf{Q} / \stackrel{0}{\simeq}$. Here $\stackrel{0}{\simeq}$ is a natural equivalence relation in $\mathbf{Q}$ given by 0-homotopies, which are filtrationpreserving homotopies with respect to skeleta. More precisely, two morphisms 
$f, g: \sigma \rightarrow \sigma^{\prime}$ are 0-homotopic if $f_{n}=g_{n}$ for $n=1$ and $n \geq 4$, and there is a $\pi_{1} f$-equivariant homomorphism $\alpha: C_{2} \rightarrow C_{2}^{\prime} \otimes C_{2}^{\prime}$ such that if $\phi: \sigma_{2} \rightarrow C_{2}$ is the natural projection, then

$$
-f_{2}(a)+g_{2}(a)=d_{3}^{\prime} \omega^{\prime} \alpha \phi(a),-f_{3}(b)+g_{3}(b)=\omega^{\prime} \alpha \phi d_{3}(b)
$$

for any $a \in \sigma_{2}$, and $b \in \sigma_{3}$. The functor $\rho$ (resp. $\sigma$ ) is full and faithful when we restrict to the full subcategory of spaces of dimension 2 (resp. 3) and take the quotient by the homotopy relation, in particular, there are natural isomorphisms $\pi_{n} \rho(X) \simeq \pi_{n} X$ (resp. $\left.\pi_{n} \sigma(X) \simeq \pi_{n} X\right)$ for $n \leq 2$ (resp. $n \leq 3$ ). Moreover, the functor $\lambda$ factors trough $\mathbf{Q} / \stackrel{0}{\simeq}$ and there is a natural equivalence $\rho=\lambda \sigma$. Furthermore, $\sigma$ and $\rho$ preserve cylinders and homotopy push-outs, hence the following result follows directly from Theorem 4.3

Corollary 4.7. For any space $X$ there is a natural isomorphism $\tilde{\Sigma} \rho(X) \simeq \sigma(\Sigma X)$.

The 2-type of a crossed complex $\rho=\left(\rho_{*}, d_{*}\right)$ is the crossed module $P_{2} \rho=$ $d_{2}: \rho_{2} / d_{3} \rho_{3} \rightarrow \rho_{1}$ and the 3 -type of a quadratic complex $\sigma=\left(\sigma_{*}, d_{*}, \omega\right)$ is the quadratic module $P_{3} \sigma$ given by

$$
C_{2} \otimes C_{2} \stackrel{\omega}{\rightarrow} \sigma_{3} / d_{4} \sigma_{4} \stackrel{d_{3}}{\rightarrow} \sigma_{2} \stackrel{d_{2}}{\rightarrow} \sigma_{1}
$$

For any space $X$ the algebraic objects $P_{2} \rho(X)$ and $P_{3} \sigma(X)$ are models of its topological 2-type $P_{2} X$ and 3-type $P_{3} X$ in the following sense: a map $f: X \rightarrow Y$ induces a weak equivalence $P_{2} \rho(f): P_{2} \rho(X) \rightarrow P_{2} \rho(Y)$ if and only if $P_{2} f: P_{2} X \rightarrow$ $P_{2} Y$ is a homotopy equivalence, and the same for $P_{3} \sigma$; see [7].

The next corollary is a direct consequence of Corollary 4.7, and shows that the suspension functor from crossed to quadratic modules defined in Proposition 3.3 is equivalent to the topological functor which sends the 2-type of $X$ to the 3 -type of $\Sigma X$.

Corollary 4.8. Given any space $X$, there is a natural isomorphism $\tilde{\Sigma} P_{2} \rho(X) \simeq$ $P_{3} \sigma(\Sigma X)$.

\section{The CO-H-STRUCTURe of A SUSPended QuAdratic COMPleX}

As in any $I$-category, suspensions in $\mathbf{Q}$ are equipped with a natural co-H-group structure given by morphisms

- the co-H-multiplication $\mu_{\sigma}: \Sigma \sigma \rightarrow \Sigma \sigma \vee \Sigma \sigma$,

- and the co-H-inversion $\nu_{\sigma}: \Sigma \sigma \rightarrow \Sigma \sigma$,

such that

$$
\left(1 \vee \mu_{\sigma}\right) \mu_{\sigma} \simeq\left(\mu_{\sigma} \vee 1\right) \mu_{\sigma},(1,0) \mu_{\sigma} \simeq 1 \simeq(0,1) \mu_{\sigma},\left(1, \nu_{\sigma}\right) \mu_{\sigma} \simeq 0 \simeq\left(\nu_{\sigma}, 1\right) \mu_{\sigma}
$$

here the symbol $\simeq$ denotes the homotopy relation. These morphisms are well defined up to homotopy; see [6], II.6. In this section we shall give explicit formulas for these morphisms. Moreover, we shall prove that the co-H-structure of $\Sigma \sigma$ is a strict cogroup structure in $\mathbf{Q}$, that is, the homotopies above are in fact equalities.

Remark 5.1. The quadratic complex of a wedge of circles $\sigma\left(\vee_{E} S^{1}\right)$ is naturally isomorphic to $\pi_{1}\left(\vee_{E} S^{1}\right)=\langle E\rangle$ concentrated in degree 1 and trivial otherwise. Therefore the canonical co-H-structure of $S^{1}$ induces the co-H-structure in $\sigma S^{1}$ 
given by

- the co-H-multiplication $\mu: \sigma S^{1} \rightarrow \sigma S^{1} \vee \sigma S^{1}$, which is $\mu_{1}:\langle e\rangle \rightarrow\left\langle e_{1}, e_{2}\right\rangle$, $\mu_{1}(e)=e_{1}+e_{2}$, in dimension 1 , here $e_{k}=i_{k} e(k=1,2)$,

- and the co-H-inversion $\nu: \sigma S^{1} \rightarrow \sigma S^{1}$, given by $\nu_{1}:\langle e\rangle \rightarrow\langle e\rangle, \nu_{1}(e)=-e$.

One readily checks that this is in fact a strict cogroup structure in $\mathbf{Q}$, that is, the homotopies in (5.A) are strict equalities in $\mathbf{Q}$.

In 7 Baues constructs a tensor product of quadratic complexes $\sigma \otimes \sigma^{\prime}$ such that $\sigma(X) \otimes \sigma(Y)$ is isomorphic to the quadratic complex of the product space $\sigma(X \times Y)$. This isomorphism is natural in $Y$ if we fix $X$ and vice versa. Moreover, there is a natural inclusion (actually a cofibration) of the coproduct of two quadratic complexes into their tensor product $\jmath: \sigma \vee \sigma^{\prime} \longmapsto \sigma \otimes \sigma^{\prime}$, which corresponds to the natural inclusion $X \vee Y \subset X \times Y$; see [7], IV.12.2 (3). Hence if we define the smash product of quadratic complexes as the cofiber of $\jmath$,

$$
\sigma \wedge \sigma^{\prime}=\sigma \otimes \sigma^{\prime} / \sigma \vee \sigma^{\prime},
$$

we get an induced isomorphism

$$
\sigma(X \wedge Y) \simeq \sigma(X) \wedge \sigma(Y) .
$$

Remark 5.2. Notice that smash products are always reduced quadratic complexes, since the natural inclusion $\jmath$ is an isomorphism in dimension 1. Moreover, the smash product is naturally distributive with respect to the coproduct

$$
\left(\sigma \wedge \sigma^{\prime \prime}\right) \vee\left(\sigma^{\prime} \wedge \sigma^{\prime \prime}\right) \simeq\left(\sigma \vee \sigma^{\prime}\right) \wedge \sigma^{\prime \prime}
$$

The isomorphism is $\left(i_{1} \wedge 1, i_{2} \wedge 1\right)$.

In the following proposition we give a further characterization of the suspension functor in $\mathbf{Q}$ which will allow us to compute explicit formulas for the co- $\mathrm{H}-$ structures.

Proposition 5.3. The suspension functor in the I-category $\mathbf{Q}$ coincides with the smash product with the quadratic complex of the circle $S^{1}$,

$$
\Sigma=\sigma S^{1} \wedge-: \mathbf{Q} \longrightarrow \mathbf{Q},
$$

up to natural equivalence. Furthermore, under this equivalence the co-H-structure of a suspension is induced by the canonical co-H-structure of $\sigma S^{1}$, that is,

$$
\mu_{\sigma}=\mu \wedge 1_{\sigma}, \quad \nu_{\sigma}=\nu \wedge 1_{\sigma},
$$

and therefore it is a strict cogroup structure in $\mathbf{Q}$.

Proof. Suppose we have checked that $\Sigma=\sigma S^{1} \wedge-, \mu_{\sigma}=\mu \wedge 1_{\sigma}$ and $\nu_{\sigma}=\nu \wedge 1_{\sigma}$. Then $\mu_{\sigma}, \nu_{\sigma}$ is a strict cogroup structure in $\mathbf{Q}$ because $\mu, \nu$ is a strict cogroup structure for $\sigma S^{1}$ and $-\wedge \sigma$ is a functor from $\mathbf{Q}$ to $\mathbf{Q}$.

If $\sigma$ is a quadratic complex with $\sigma_{n}=0$ for $n>4$, one can construct a 4dimensional space $X$ with $\sigma=\sigma X$ by using [7, IV.7.5, compare with IV.8.5 in the same reference. Since $\sigma$ preserves cylinders and its structural maps, it also preserves suspensions and its co-H-structures. Moreover, it is well known that $S^{1} \wedge-$ coincides with the suspension functor in the category of spaces, and the canonical co-H-structure of a suspended space is induced by the usual co-H-structure of $S^{1}$. Therefore, the proposition is true for a quadratic complex $\sigma$ with $\sigma_{n}=0$ for $n>4$. For a general quadratic complex it is a consequence of the previous 
particular case and the fact that chain complexes admit a unique co-H-structure up to homotopy; see the next lemma.

Lemma 5.4. A chain complex (of abelian groups) admits a unique co-H-structure up to homotopy.

Proof. This category of chain complexes is an additive category. We can define a co-H-structure on a chain complex $C_{*}$ by $\mu=i_{1}+i_{2}$ and $\nu=-1$. Consider another co-H-structure given by $\mu^{\prime}$ and $\nu^{\prime}$. There are chain homotopies $\left\{\alpha_{n}\right\}_{n \in \mathbb{Z}}:(1,0) \mu^{\prime} \simeq$ $1,\left\{\beta_{n}\right\}_{n \in \mathbb{Z}}:(0,1) \mu^{\prime} \simeq 1$. One readily checks that $\left\{i_{1} \alpha_{n}+i_{2} \beta_{n}\right\}_{n \in \mathbb{Z}}$ is a chain homotopy $\mu^{\prime} \simeq \mu$. Similarly, if $\left\{\gamma_{n}\right\}_{n \in \mathbb{Z}}:\left(1, \nu^{\prime}\right) \mu \simeq 0$ is a chain homotopy, then $\left\{\gamma_{n}\right\}_{n \in \mathbb{Z}}$ is a chain homotopy $\nu^{\prime} \simeq \nu$ as well.

A direct consequence of Proposition 5.3 is the following.

Corollary 5.5. The morphism set $\mathbf{Q}\left(\Sigma \sigma, \sigma^{\prime}\right)$ has a group structure such that the natural projection onto the group of homotopy classes $\mathbf{Q}\left(\Sigma \sigma, \sigma^{\prime}\right) \rightarrow\left[\Sigma \sigma, \sigma^{\prime}\right]$ is a homomorphism.

In order to compute the cogroup structure of a suspended quadratic complex we introduce the following notation. Given a homomorphism $\varphi:\langle E\rangle^{\text {nil }} \rightarrow\langle F\rangle^{\text {nil }}, \nabla \varphi$ is the unique homomorphism

$$
\nabla \varphi: \mathbb{Z}\langle E\rangle \rightarrow \mathbb{Z}\langle F\rangle^{\otimes^{2}}
$$

such that $s \imath(\nabla \varphi) p=\left(i_{2} \mid i_{1}\right)_{\varphi} ;$ moreover,

$$
\Delta: \mathbb{Z}\langle E\rangle \rightarrow \mathbb{Z}\langle E\rangle^{\otimes^{2}}
$$

is the non-natural diagonal homomorphism defined on generators by $\Delta(a)=a \otimes a$, $(a \in E)$.

The comultiplication of the suspension of a quadratic complex $\sigma$ such that $\bar{\varphi}:\left\langle E_{2}\right\rangle \rightarrow\left\langle E_{1}\right\rangle$ is the basis of the nil(2)-module $d_{2}: \sigma_{2} \rightarrow \sigma_{1}$, is completely determined by the formulas of the following theorem, where $\varphi=\bar{\varphi}^{\text {nil }}$. The morphisms $r$ and $\omega$ are the structural morphisms of any totally free reduced quadratic module in the central push-out diagram (3.C).

Theorem 5.6. The comultiplication $\mu_{\sigma}$ in $\mathbf{Q}$ verifies the following equalities:

(1) $\left(\mu_{\sigma}\right)_{n}=i_{1}+i_{2}$ if $n \neq 3$,

(2) $\left(\mu_{\sigma}\right)_{3} r=r\left(i_{1}+i_{2}\right)+\omega\left(i_{2} \otimes i_{1}\right)\left(\nabla \varphi+\Delta \varphi^{a b}\right) p$,

(3) $\left(\mu_{\sigma}\right)_{3} \omega=\omega\left(i_{1}+i_{2}\right)^{\otimes^{2}}$.

Before proving this result we shall state some consequences.

Corollary 5.7. The group structure in $\mathbf{Q}\left(\Sigma \sigma, \sigma^{\prime}\right)$ is given by the following formulas $\left(f, g \in \mathbf{Q}\left(\Sigma \sigma, \sigma^{\prime}\right)\right)$ :

(1) $(f+g)_{n}=f_{n}+g_{n}$ if $n \neq 3$,

(2) $(f+g)_{3} r=f_{3} r+g_{3} r+\omega\left(g_{2}^{a b} \otimes f_{2}^{a b}\right)\left(\nabla \varphi+\Delta \varphi^{a b}\right) p$,

(3) $(f+g)_{3} \omega=\omega\left(f_{2}^{a b}+g_{2}^{a b}\right)^{\otimes^{2}}$,

and

(4) $(-f)_{n}=-f_{n}$ if $n \neq 3$,

(5) $(-f)_{3} r=-f_{3} r+\omega\left(f_{2}^{a b} \otimes f_{2}^{a b}\right)\left(\nabla \varphi+\Delta \varphi^{a b}\right) p$,

(6) $(-f)_{3} \omega=\omega\left(f_{2}^{a b} \otimes f_{2}^{a b}\right)$. 
Proof. Recall that $\sigma_{3}^{\prime}$ and $\operatorname{Ker}\left[d_{2}: \sigma_{2}^{\prime} \rightarrow \sigma_{1}^{\prime}\right]$ are nil(2)-groups (see (3.1) and (3.2)), so the formulas above make sense. Equality (1) is trivial, (3) follows from (1), and (4), (5), (6) can be obtained from (1), (2), (3) by imposing $f+(-f)=0$. Finally, (2) follows from the equalities:

$$
\begin{aligned}
(f+g)_{3} r=(f, g)_{3}\left(\mu_{\sigma}\right)_{3} r= & (f, g)_{3} r\left(i_{1}+i_{2}\right)+(f, g)_{3} \omega\left(i_{2} \otimes i_{1}\right)\left(\nabla \varphi+\Delta \varphi^{a b}\right) p \\
= & \left(f_{3} r, g_{3} r\right)\left(i_{1}+i_{2}\right) \\
& +\omega\left(f_{2}^{a b}, g_{2}^{a b}\right)\left(i_{2} \otimes i_{1}\right)\left(\nabla \varphi+\Delta \varphi^{a b}\right) p \\
= & f_{3} r+g_{3} r+\omega\left(g_{2}^{a b} \otimes f_{2}^{a b}\right)\left(\nabla \varphi+\Delta \varphi^{a b}\right) p .
\end{aligned}
$$

In (a) we use that the basis of the coproduct of totally free quadratic complexes is the coproduct of the basis.

Since $\nu_{\sigma}=-1 \in \mathbf{Q}(\Sigma \sigma, \Sigma \sigma)$, we also derive the following.

Corollary 5.8. The coinversion $\nu_{\sigma}$ is determined by the following equalities:

(1) $\left(\nu_{\sigma}\right)_{n}=-1$ if $n \neq 3$,

(2) $\left(\nu_{\sigma}\right)_{3} r=-r+\omega\left(\nabla \varphi+\Delta \varphi^{a b}\right) p$,

(3) $\left(\nu_{\sigma}\right)_{3} \omega=\omega$.

In order to prove Theorem [5.6 we first give some technical results.

Lemma 5.9. If $a_{i} \in E, \epsilon_{i} \in \mathbb{Z}$ and $x=\epsilon_{1} a_{1}+\cdots+\epsilon_{n} a_{n} \in\langle E\rangle^{n i l}$, then the following equalities hold in $\langle E\rangle^{\text {nil }} \vee\langle E\rangle^{\text {nil }}$ :

$$
\begin{aligned}
\left(i_{2}+i_{1}\right) x-i_{1} x-i_{2} x= & \sum_{1 \leq i<j \leq n} \epsilon_{i} \epsilon_{j}\left[i_{1} a_{i}, i_{2} a_{j}\right] \\
& +\sum_{i=1}^{n}\left(\begin{array}{c}
\left|\epsilon_{i}\right| \\
2
\end{array}\right)\left[i_{1} a_{i}, i_{2} a_{i}\right]-\sum_{\epsilon_{i}<0} \epsilon_{i}\left[i_{1} a_{i}, i_{2} a_{i}\right] \\
= & s \imath\left(\sum_{1 \leq i<j \leq n} \epsilon_{i} \epsilon_{j} a_{i} \otimes a_{j}\right. \\
& \left.+\sum_{i=1}^{n}\left(\begin{array}{c}
\left|\epsilon_{i}\right| \\
2
\end{array}\right) a_{i} \otimes a_{i}-\sum_{\epsilon_{i}<0} \epsilon_{i} a_{i} \otimes a_{i}\right)
\end{aligned}
$$

Here $\left(\begin{array}{c}m \\ 2\end{array}\right)=\frac{m(m-1)}{2}$ for any $m \geq 0$.

Proof. The second equality is obvious. We proceed by induction on the number of summands. Suppose $n=1, m>0$ and $a=a_{1}$. The result is trivial for $\epsilon_{1}=m=1$. If $\epsilon_{1}=m+1$ and it is true for $\epsilon_{1}=m$, then

$$
\begin{aligned}
\left(i_{2}+i_{1}\right) x-i_{1} x-i_{2} x & =(m+1)\left(i_{2} a+i_{1} a\right)-(m+1) i_{1} a-(m+1) i_{2} a \\
& =m\left(i_{2} a+i_{1} a\right)+i_{2} a+i_{1} a-i_{1} a-m i_{1} a-i_{2} a-m i_{2} a \\
& =m\left(i_{2} a+i_{1} a\right)-m i_{1} a+\left[-m i_{1} a,-i_{2} a\right]-m i_{2} a \\
& =m\left(i_{2} a+i_{1} a\right)-m i_{1} a-m i_{2} a+\left[m i_{1} a, i_{2} a\right] \\
& =\left(\begin{array}{c}
m \\
2
\end{array}\right)\left[i_{1} a, i_{2} a\right]+m\left[i_{1} a, i_{2} a\right] \\
& =\left(\begin{array}{c}
m+1 \\
2
\end{array}\right)\left[i_{1} a, i_{2} a\right] .
\end{aligned}
$$


The induction hypothesis is applied in (a). If $\epsilon_{1}=-m$ for some $m>0$, then

$$
\begin{aligned}
\left(i_{2}+i_{1}\right)(-m a)-i_{1}(-m a)-i_{2}(-m a) & =-m\left(i_{2} a+i_{1} a\right)+m i_{1} a+m i_{2} a \\
& =m\left(-i_{1} a-i_{2} a\right)+m i_{1} a+m i_{2} a .
\end{aligned}
$$

Now we check by induction on $m$ that this is equal to $\left(\begin{array}{c}m \\ 2\end{array}\right)\left[i_{1} a, i_{2} a\right]+m\left[i_{1} a, i_{2} a\right]$. It is clear for $m=1$. If it is true for $m$, then

$$
\begin{aligned}
(m+1)\left(-i_{1} a-i_{2} a\right) & \\
+(m+1) i_{1} a+(m+1) i_{2} a= & m\left(-i_{1} a-i_{2} a\right)-i_{1} a-i_{2} a \\
& +i_{1} a+m i_{1} a+i_{2} a+m i_{2} a \\
= & m\left(-i_{1} a-i_{2} a\right)+\left[i_{1} a, i_{2} a\right] \\
& +\left[i_{2} a,-m i_{1} a\right]+m i_{1} a+m i_{2} a \\
= & m\left(-i_{1} a-i_{2} a\right)+m i_{1} a+m i_{2} a+(m+1)\left[i_{1} a, i_{2} a\right] \\
= & \left(\begin{array}{c}
m \\
2
\end{array}\right)\left[i_{1} a, i_{2} a\right]+m\left[i_{1} a, i_{2} a\right]+(m+1)\left[i_{1} a, i_{2} a\right] \\
(\mathrm{b}) \quad & \left(\begin{array}{c}
m+1 \\
2
\end{array}\right)\left[i_{1} a, i_{2} a\right]+(m+1)\left[i_{1} a, i_{2} a\right] .
\end{aligned}
$$

The induction step is given in (b).

Suppose now that the lemma is true for $\leq n$ summands $(n \geq 1)$. Let us check it for $n+1$ summands. For this, we set $y=\epsilon_{1} a_{1}+\cdots+\epsilon_{n} a_{n}, \epsilon=\epsilon_{n+1}$ and $a=a_{n+1}$. Then

$$
\begin{aligned}
\left(i_{2}+i_{1}\right) x-i_{1} x-i_{2} x= & \left(i_{2}+i_{1}\right) y+\left(i_{2}+i_{1}\right)(\epsilon a)-i_{1}(\epsilon a)-i_{1} y-i_{2}(\epsilon a)-i_{2} y \\
= & \left(i_{2}+i_{1}\right) y+\left(i_{2}+i_{1}\right)(\epsilon a)-i_{1}(\epsilon a)-i_{2}(\epsilon a) \\
& +\left[-i_{2}(\epsilon a), i_{1} y\right]-i_{1} y-i_{2} y \\
= & \left(i_{2}+i_{1}\right) y-i_{1} y-i_{2} y \\
& +\left(i_{2}+i_{1}\right)(\epsilon a)-i_{1}(\epsilon a)-i_{2}(\epsilon a)+\left[i_{1} y, i_{2}(\epsilon a)\right] \\
(\mathrm{c}) \quad & \sum_{1 \leq i<j \leq n} \epsilon_{i} \epsilon_{j}\left[i_{1} a_{i}, i_{2} a_{j}\right]+\sum_{i=1}^{n}\left(\begin{array}{c}
\left|\epsilon_{i}\right| \\
2
\end{array}\right)\left[i_{1} a_{i}, i_{2} a_{i}\right] \\
& -\sum_{\epsilon_{i}<0, i \leq n} \epsilon_{i}\left[i_{1} a_{i}, i_{2} a_{i}\right]+\left(\begin{array}{c}
|\epsilon| \\
2
\end{array}\right)\left[i_{1} a, i_{2} a\right] \\
& +\operatorname{sign}(\epsilon) \epsilon\left[i_{1} a, i_{2} a\right]+\sum_{i=1}^{n} \epsilon_{i} \epsilon\left[i_{1} a_{i}, i_{2} a\right] \\
& \sum_{1 \leq i<j \leq n+1} \epsilon_{i} \epsilon_{j}\left[i_{1} a_{i}, i_{2} a_{j}\right]+\sum_{i=1}^{n+1}\left(\begin{array}{c}
\left|\epsilon_{i}\right| \\
2
\end{array}\right)\left[i_{1} a_{i}, i_{2} a_{i}\right] \\
& -\sum_{\epsilon_{i}<0} \epsilon_{i}\left[i_{1} a_{i}, i_{2} a_{i}\right] .
\end{aligned}
$$

Here $\operatorname{sign}(\epsilon)$ is -1 if $\epsilon<0$ and 0 otherwise. In (c) we use that $\left(i_{2}+i_{1}\right)(\epsilon a)-i_{1}(\epsilon a)-$ $i_{2}(\epsilon a)$ belongs to the commutator subgroup, by the case $n=1$, and commutators are central in any nil(2)-group. We apply the induction hypothesis in (d). Now the proof is finished.

Lemma 5.10. For any $\varphi \in$ nil we have $\nabla \varphi+\nabla(-\varphi)=\left(\varphi^{a b} \otimes \varphi^{a b}\right) \Delta$. 
Proof. In fact, it follows from Lemma 5.9 that if $a_{i} \in E, \epsilon_{i} \in \mathbb{Z}$ and $x=\epsilon_{1} a_{1}+$ $\cdots+\epsilon_{n} a_{n} \in\langle E\rangle^{n i l}$, then

$$
\begin{aligned}
\left(i_{2}+i_{1}\right) x-i_{1} x-i_{2} x & \\
+\left(i_{2}+i_{1}\right)(-x)-i_{1}(-x)-i_{2}(-x) & =\sum_{i, j=1}^{n} \epsilon_{i} \epsilon_{j}\left[i_{1} a_{i}, i_{2} a_{j}\right]=s \imath(p(x) \otimes p(x)),
\end{aligned}
$$

hence the lemma holds.

Now we are ready to prove Theorem 5.6 .

Proof of Theorem 5.6. The first formula can be easily checked by using the description of the suspension functor in $\mathbf{Q}$ and the cogroup structure of a suspension given in Proposition [5.3, together with the formulas in [7, IV.12.10, IV.12.3 (3) and IV.12.3 (5). The third one is a consequence of (1) for $n=2$.

In order to prove (2) consider $b \in E$ with $d_{2}(b)=\bar{\varphi}(b)=\epsilon_{1} a_{1}+\cdots \epsilon_{n} a_{n}$, for some $a_{i} \in F, \epsilon_{i} \in \mathbb{Z}$. Then $\left(\mu_{\sigma}\right)_{3} r(b)$ is the projection of $\left(e_{1}+e_{2}\right) \overline{\bar{\otimes}} b \in\left(\left(\sigma S^{1} \vee \sigma S^{1}\right) \otimes \sigma\right)_{3}$ to the quotient $(\Sigma \sigma \vee \Sigma \sigma)_{3}=\left(\left(\sigma S^{1} \vee \sigma S^{1}\right) \otimes \sigma /\left(\sigma S^{1} \vee \sigma S^{1}\right) \vee \sigma\right)_{3}$; see [7], IV.12.10 and IV.12.5 (2). In the following equations the formulas are in $\left(\left(\sigma S^{1} \vee \sigma S^{1}\right) \otimes \sigma\right)_{3}$ and the symbol $\approx$ means that they coincide when projecting onto $(\Sigma \sigma \vee \Sigma \sigma)_{3}=$ $\left(\left(\sigma S^{1} \vee \sigma S^{1}\right) \otimes \sigma /\left(\sigma S^{1} \vee \sigma S^{1}\right) \vee \sigma\right)_{3}$

(a) $\left(e_{1}+e_{2}\right) \overline{\bar{\otimes}} b=-S_{2}^{e_{1}+e_{2}}(b)$

$$
\begin{aligned}
& =\omega \Theta\left(e_{1}+e_{2}, \bar{\varphi}(b)\right)+S(b)\left(e_{1}+e_{2}\right) \\
& \approx-\omega \widetilde{T}\left(\vartheta^{\sigma S^{1} \vee \sigma S^{1}}\left(e_{1}+e_{2}\right) \otimes \vartheta^{\sigma} \bar{\varphi}(b)\right)+e_{2} \times b+e_{1} \times b .
\end{aligned}
$$

Here $\widetilde{T}$ is the same as $T$ in [7, IV.12.4(3). We have changed the name of this homomorphism because in this paper $T$ is the interchange of factors in the tensor square; see (2.2). We have used [7], IV.12.5 (13) for (a), IV.12.5 (12) for (b), and IV.12.4 (6) together with IV.12.5 (9) for (c). Moreover, by [7, IV.12.4 (1), we have that $\vartheta^{\sigma}(0)=\vartheta^{\sigma}(0+0) \approx \vartheta^{\sigma}(0)+\vartheta^{\sigma}(0)$, and given $a \in F$,

$$
0 \approx \vartheta^{\sigma}(0)=\vartheta^{\sigma}(a+(-a)) \approx \vartheta^{\sigma}(a)+\vartheta^{\sigma}(-a)-a \otimes a=\vartheta^{\sigma}(-a)-a \otimes a,
$$

so $\vartheta^{\sigma}(-a) \approx a \otimes a$. By using this relation one can check inductively, as in the proof of Lemma 5.9, that the following relation holds:

$$
\vartheta^{\sigma} \bar{\varphi}(b) \approx \sum_{1 \leq i<j \leq n} \epsilon_{i} \epsilon_{j} a_{i} \otimes a_{j}+\sum_{i=1}^{n}\left(\begin{array}{c}
\left|\epsilon_{i}\right| \\
2
\end{array}\right) a_{i} \otimes a_{i}-\sum_{\epsilon_{1}<0} \epsilon_{i} a_{i} \otimes a_{i}
$$

and thus by using [7], IV.12.4 (3), and the fact that $\omega$ is central we see that

$$
\begin{aligned}
(\mathrm{c}) \approx & e_{2} \times b+e_{1} \times b-\sum_{1 \leq i<j \leq n} \epsilon_{i} \epsilon_{j} \omega\left(e_{2} \otimes a_{j} \otimes e_{1} \otimes a_{i}\right) \\
& -\sum_{i=1}^{n}\left(\begin{array}{c}
\left|\epsilon_{i}\right| \\
2
\end{array}\right) \omega\left(e_{2} \otimes a_{i} \otimes e_{1} \otimes a_{i}\right)+\sum_{\epsilon_{i}<0} \epsilon_{i} \omega\left(e_{2} \otimes a_{i} \otimes e_{1} \otimes a_{i}\right) .
\end{aligned}
$$


This proves, by projecting onto $(\Sigma \sigma \vee \Sigma \sigma)_{3}$, that

$$
\begin{aligned}
\left(\mu_{\sigma}\right)_{3} r(b)= & r i_{2}(b)+r i_{1}(b)-\omega\left(i_{2} \otimes i_{1}\right)\left(\sum_{1 \leq j<i \leq n} \epsilon_{i} \epsilon_{j} a_{i} \otimes a_{j}\right. \\
& \left.+\sum_{i=1}^{n}\left(\begin{array}{c}
\left|\epsilon_{i}\right| \\
2
\end{array}\right) a_{i} \otimes a_{i}-\sum_{\epsilon_{1}<0} \epsilon_{i} a_{i} \otimes a_{i}\right) \\
= & r i_{2}(b)+r i_{1}(b)-\omega\left(i_{2} \otimes i_{1}\right)\left(\nabla(-\varphi)(b)-\Delta \varphi^{a b}(b)\right) .
\end{aligned}
$$

In (d) we use Lemma 5.9 By Lemma 5.10 this implies that

$$
\left(\mu_{\sigma}\right)_{3} r=r\left(i_{2}+i_{1}\right)+\omega\left(i_{2} \otimes i_{1}\right)\left(\nabla \varphi-\left(\varphi^{a b} \otimes \varphi^{a b}\right) \Delta+\Delta \varphi^{a b}\right) p .
$$

Recall that the basis of the low-dimensional reduced quadratic module of the coproduct $\Sigma \sigma \vee \Sigma \sigma$ is $(-\varphi) \vee(-\varphi) \in$ nil, hence by using (3.C) we obtain

$$
\omega\left(i_{2} \otimes i_{1}\right)\left(\varphi^{a b} \otimes \varphi^{a b}\right) \Delta p=\omega\left(\varphi^{a b} \oplus \varphi^{a b}\right)^{\otimes^{2}}\left(i_{2} \otimes i_{1}\right) \Delta p=r s q\left(i_{2} \otimes i_{1}\right) \Delta p .
$$

Furthermore, for any $b \in E$ we have that $r s q\left(i_{2} \otimes i_{1}\right) \Delta p(b)=r\left[i_{2} b, i_{1} b\right]$ and therefore

$$
(e)=r\left(i_{1}+i_{2}\right)+\omega\left(i_{2} \otimes i_{1}\right)\left(\nabla \varphi+\Delta \varphi^{a b}\right) p .
$$

\section{The natural homomorphism $H_{2} X \rightarrow \wedge^{2} H_{1} X$}

The Pontrjagin product in the homology of an abelian group $A$ induces an isomorphism $\wedge^{2} A \simeq H_{2} A$; see [13]. In [15] it is proven that the James-Hopf invariant $\gamma_{2}: \pi_{3} \Sigma K(A, 1) \rightarrow A^{\otimes^{2}}$ is an isomorphism; moreover, the Hurewicz homomorphism $h_{3}$ corresponds with the natural projection $q$ in the following diagram:

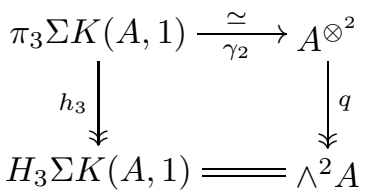

Given any space $X$, consider the natural homotopy class $h: X \rightarrow K\left(H_{1} X, 1\right)$ such that $\pi_{1} h=h_{1}: \pi_{1} X \rightarrow H_{1} X$ is the Hurewicz homomorphism. The naturality of the James-Hopf invariant $\gamma_{2}$, the Hurewicz homomorphism, the suspension isomorphism in homology, and (6.A) imply that the following diagram also commutes

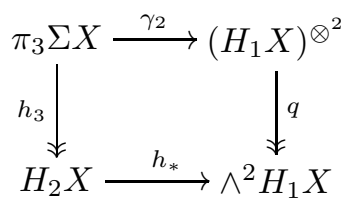

In this section we shall compute the natural homomorphism $h_{*}$ by using the results of Section 5. The results concerning the co-H-structure of a suspension are useful because of the existent relation between the James-Hopf invariant and this co-H-structure; see (6.C) below. 
We can suppose, without loss of generality, that the 2-skeleton $X^{2}$ of the space $X$ is the mapping cone of a based map between wedges of circles

$$
f: \vee_{E_{2}} S^{1} \longrightarrow \vee_{E_{1}} S^{1} .
$$

This map carries the same homotopical information as the induced homomorphism in $\pi_{1}$,

$$
\bar{\varphi}=\pi_{1} f:\left\langle E_{2}\right\rangle \longrightarrow\left\langle E_{1}\right\rangle,
$$

which is the basis of the low-dimensional nil(2)-module of the quadratic complex $\sigma(X)$.

If we write $C_{*} X$ for the (reduced) cellular chain complex of $X$, it is clear that $E_{i} \subset C_{i} X$ are bases $(i=1,2)$; moreover, $\bar{\varphi}^{a b}=d_{2}: C_{2} X \rightarrow C_{1} X$.

Theorem 6.1. Choose a basis of 2-cycles $Z_{2} \subset \operatorname{Ker} d_{2}$ and a homomorphism $\psi:\left\langle Z_{2}\right\rangle^{n i l} \rightarrow\left\langle E_{2}\right\rangle^{n i l}$ such that $\psi^{a b}:$ Ker $d_{2} \hookrightarrow C_{2} X$ is the inclusion. The natural homomorphism $h_{*}: H_{2} X \rightarrow \wedge^{2} H_{1} X$ is induced by the unique homomorphism $\alpha$ : Ker $d_{2} \rightarrow \wedge^{2} C_{1} X$ verifying $\varphi \psi=s \alpha p$, where $\varphi=\bar{\varphi}^{\text {nil }}$, and $p$ and $s$ are part of the central extension in (2.G).

Before beginning with the proof of this result we shall give some homotopical considerations. The following natural identifications are obtained by using Hurewicz's theorem, the suspension isomorphism in homology and Künneth's formula,

$$
\pi_{3} \Sigma X \wedge X=H_{3} \Sigma X \wedge X=H_{2} X \wedge X=\left(H_{1} X\right)^{\otimes^{2}} .
$$

If $h_{1}: \pi_{1} X \rightarrow H_{1} X$ is the Hurewicz homomorphism, then the natural identification $\left(H_{1} X\right)^{\otimes^{2}}=\pi_{3} \Sigma X \wedge X$ sends a generator $h_{1}(f) \otimes h_{1}(g)$ to the homotopy class of the map $\Sigma(f \wedge g): S^{3}=\Sigma S^{1} \wedge S^{1} \rightarrow \Sigma X \wedge X$; compare Section 5 in 12 and 1] , 6.3.16. By using the naturality properties of the Whitehead product element $\left[i_{1}, i_{2}\right]_{Y} \in[\Sigma Y \wedge Y, \Sigma(Y \vee Y)]$ (see [8]), we observe that the following diagram commutes:

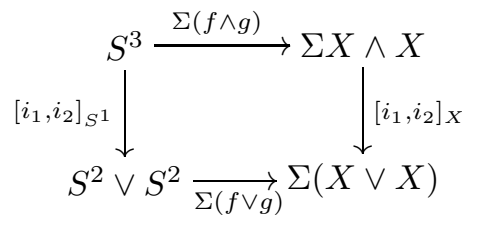

Hence the homotopy left distributivity formula in [8], A.10.2 (b), proves that the following diagram commutes:

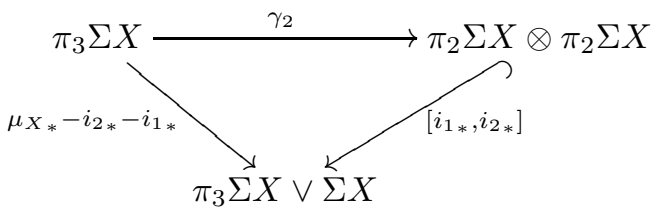

Here the bracket [, ] is the usual Whitehead product operation in homotopy groups, induced by $\left[i_{1}, i_{2}\right]_{S^{1}}$, and $\left[i_{1 *}, i_{2 *}\right](f \otimes g)=\left[i_{1} f, i_{2} g\right],\left(f, g \in \pi_{2} \Sigma X\right)$. It is a wellknown fact that $\left[i_{1_{*}}, i_{2 *}\right]$ is a splitting injection whose cokernel is $\pi_{3} \Sigma X \oplus \pi_{3} \Sigma X$.

Diagram (6.C) and formulas in Theorem [5.6 will help us to compute the JamesHopf invariant $\gamma_{2}$ in certain cases, which allow us to prove Theorem 6.1 by using the commutativity of (6.B).

We also need the translation of the Whitehead product operation and the Hurewicz homomorphism $h_{3}$ to the language of quadratic complexes. For this, note that, 
when $\sigma$ is a reduced quadratic complex, the second homotopy group $\pi_{2} \sigma$ is a quotient of $\sigma_{2}$ and the natural projection $\sigma_{2} \rightarrow \pi_{2} \sigma$ factors through the abelianization $\sigma_{2} \rightarrow \sigma_{2}^{a b}=C_{2}$.

The Whitehead product homomorphism $\left[i_{1 *}, i_{2 *}\right]$ for a suspended quadratic complex $\Sigma \sigma$ can be computed by passing to the quotient the following homomorphism

$$
\omega\left(i_{1} \otimes i_{2}+i_{2} \otimes i_{1} T\right):(\Sigma \sigma)_{2}^{a b} \otimes(\Sigma \sigma)_{2}^{a b} \rightarrow \operatorname{Ker} d_{3} \subset(\Sigma \sigma \vee \Sigma \sigma)_{3},
$$

compare IV.3.7 and I.4.4 in 7]. Moreover, if $\sigma=\sigma(X)$, then $C_{*} \Sigma X$ is $\lambda \Sigma \sigma$. This crossed complex is in fact a chain complex of free abelian groups because a suspended crossed complex is reduced. There is a natural projection of chain complexes of groups $\Sigma \sigma \rightarrow \lambda \Sigma \sigma=C_{*} \Sigma X$; in fact, for any quadratic complex $\sigma$ one can easily construct a canonical projection $\sigma \rightarrow \lambda \sigma$ from the construction of $\lambda$; see Section 4 or [7, IV.3.3. The Hurewicz homomorphism $h_{3}$ coincides with the morphism induced by this projection in the third homology; see IV.C.10 and IV.3.7 in [7].

Proof of Theorem 6.1. Consider $\sigma=\sigma(X)$. In order to carry out the necessary computations we choose a total ordering $\preceq$ in $E_{1}$ and define the homomorphism $\eta: \wedge^{2} \mathbb{Z}\left\langle E_{1}\right\rangle \rightarrow \mathbb{Z}\left\langle E_{1}\right\rangle^{\otimes^{2}}$ as $\eta\left(e_{1} \wedge e_{2}\right)=e_{1} \otimes e_{2}\left(e_{1} \prec e_{2} \in E_{1}\right)$. This homomorphism is a splitting of $q$ since it satisfies $q \eta=1$.

Let $\beta$ : Ker $d_{2} \rightarrow \wedge^{2} C_{1} X$ be the unique homomorphism satisfying $(-\varphi) \psi=s \beta_{p}$; by $(2.3)$ (iii)

$$
\begin{aligned}
s(\alpha+\beta) p & =(\varphi \mid \varphi)_{\psi} \\
& =(\varphi+\varphi) \psi-\varphi \psi-\varphi \psi \\
& =(\varphi, \varphi)\left(\left(i_{2}+i_{1}\right) \psi-i_{1} \psi-i_{2} \psi\right) \\
& =(\varphi, \varphi) s \iota(\nabla \psi) p \\
& =s\left(\wedge^{2} \varphi^{a b} q(\nabla \psi) p\right.
\end{aligned}
$$

so $\alpha+\beta=\left(\wedge^{2} d_{2}\right) q(\nabla \psi)$. In the last equality we use that, given $a, b \in E_{2}$,

$$
\begin{aligned}
(\varphi, \varphi) s \iota(a \otimes b) & =(\varphi, \varphi) s\left(i_{1} a \wedge i_{2} b\right)=(\varphi, \varphi)\left[i_{1} a, i_{2} b\right] \\
& =[\varphi(a), \varphi(b)]=s\left(\wedge^{2} \varphi^{a b}\right) q(a \otimes b) .
\end{aligned}
$$

The image of the homomorphism

$$
r \psi-\omega \eta \beta p:\left\langle Z_{2}\right\rangle^{n i l} \rightarrow(\Sigma \sigma)_{3}
$$

lies on Ker $d_{3}$, since, by Lemma 4.6, $d_{3}(r \psi-\omega \eta \beta p)=(-\varphi) \psi-s q \eta \beta p=s \beta p-s \beta p=$ 0 .

Let us begin with the proof of the statement of the theorem. Given a 2-cycle $x \in \mathbb{Z}\left\langle Z_{2}\right\rangle$, if $\bar{x} \in\left\langle Z_{2}\right\rangle^{\text {nil }}$ is an element such that $p(\bar{x})=x$, then

$$
y=(r \psi-\omega \eta \beta p)(\bar{x}) \in(\Sigma \sigma)_{3}
$$

represents an element of $\pi_{3} \Sigma X$ such that $h_{3}\{y\}=\{x\}$, here $\{\cdot\}$ denotes the equivalence class in the corresponding quotient group, so by (6.C),

$$
\left(\mu_{\sigma}\right)_{3}(y)-\left(i_{2}\right)_{3}(y)-\left(i_{1}\right)_{3}(y) \in(\Sigma \sigma \vee \Sigma \sigma)_{3}
$$

represents $\left[i_{1 *}, i_{2 *}\right] \gamma_{2}\{y\} \in \pi_{3}(\Sigma X \vee \Sigma X)$.

Let us compute this element and $\gamma_{2}\{y\}$. For this recall that $(-\varphi) \vee(-\varphi) \in$ nil is the basis of the low-dimensional reduced quadratic module of $\Sigma \sigma \vee \Sigma \sigma$, and the 
inclusions of the factors of the coproduct $i_{k}: \Sigma \sigma \rightarrow \Sigma \sigma \vee \Sigma \sigma(k=1,2)$ satisfy $\left(i_{k}\right)_{n}=i_{k}(n \neq 3)$ and $\left(i_{k}\right)_{3} r=r i_{k}$.

(a) $\quad\left(\mu_{\sigma}\right)_{3}(r \psi-\omega \eta \beta p)=r\left(i_{1}+i_{2}\right) \psi+\omega\left(i_{2} \otimes i_{1}\right)\left(\nabla \varphi+\Delta \varphi^{a b}\right) p \psi$

$$
-\omega\left(i_{1}+i_{2}\right)^{\otimes^{2}} \eta \beta p
$$

$$
\left(i_{1}\right)_{3}(r \psi-\omega \eta \beta p)=r i_{1} \psi-\omega\left(i_{1} \otimes i_{1}\right) \eta \beta p
$$

$$
\begin{aligned}
\left(i_{2}\right)_{3}(r \psi-\omega \eta \beta p) & =r i_{2} \psi-\omega\left(i_{2} \otimes i_{2}\right) \eta \beta p, \\
(\mathrm{a})-(\mathrm{c})-(\mathrm{b}) & =r\left(i_{1} \mid i_{2}\right)_{\psi}+\omega\left(i_{2} \otimes i_{1}\right)\left(\nabla \varphi+\Delta \varphi^{a b}\right) p \psi
\end{aligned}
$$$$
-\omega\left(i_{1} \otimes i_{2}+i_{2} \otimes i_{1}\right) \eta \beta p
$$

$$
r\left(i_{1} \mid i_{2}\right)_{\psi}=r\left(i_{2}, i_{1}\right)\left(i_{2} \mid i_{1}\right)_{\psi}
$$$$
=r\left(i_{2}, i_{1}\right) s \imath(\nabla \psi) p
$$$$
=r s \wedge^{2}\left(i_{2}, i_{1}\right) \imath(\nabla \psi) p
$$$$
=r s q\left(i_{2} \otimes i_{1}\right)(\nabla \psi) p
$$$$
=\omega\left(\varphi^{a b} \oplus \varphi^{a b}\right)^{\otimes^{2}}\left(i_{2} \otimes i_{1}\right)(\nabla \psi) p
$$$$
=\omega\left(i_{2} \otimes i_{1}\right)\left(\varphi^{a b} \otimes \varphi^{a b}\right)(\nabla \psi) p .
$$

Here we use that $\omega$ is central. Moreover, in (a) we apply Theorem 5.6 in (f) the naturality of (2.G), in (h) (3.C), and in (g) we use that for any $a, b \in C_{2} X$,

$$
\wedge^{2}\left(i_{2}, i_{1}\right) \imath(a \otimes b)=\wedge^{2}\left(i_{2}, i_{1}\right)\left(i_{1} a \wedge i_{2} b\right)=i_{2} a \wedge i_{1} b=q\left(i_{2} \otimes i_{1}\right)(a \otimes b) .
$$

Furthermore, $\varphi^{a b} p \psi=\varphi^{a b} \psi^{a b} p=0 p=0$, therefore by (e) we get

$$
\begin{aligned}
(\mathrm{d})= & -\omega\left(i_{1} \otimes i_{2}\right) \eta \beta p \\
& -\omega\left(i_{2} \otimes i_{1}\right)\left(\eta \beta-(\nabla \varphi) \psi^{a b}-\left(\varphi^{a b} \otimes \varphi^{a b}\right)(\nabla \psi)\right) p .
\end{aligned}
$$

We have the following equalities:

$$
\begin{aligned}
s \imath(\nabla \varphi) \psi^{a b} p & =\left(\left(i_{2}+i_{1}\right) \varphi-i_{1} \varphi-i_{2} \varphi\right) \psi \\
& =\left(i_{2}+i_{1}\right) \varphi \psi-\left(i_{2} \varphi+i_{1} \varphi\right) \psi, \\
s \imath\left(\varphi^{a b} \otimes \varphi^{a b}\right)(\nabla \psi) p & =s \wedge^{2}\left(\varphi^{a b} \oplus \varphi^{a b}\right) \imath(\nabla \psi) p \\
& =(\varphi \vee \varphi) s \imath(\nabla \psi) p \\
& =(\varphi \vee \varphi)\left(\left(i_{2}+i_{1}\right) \psi-i_{1} \psi-i_{2} \psi\right) \\
& =\left(i_{2} \varphi+i_{1} \varphi\right) \psi-i_{1} \varphi \psi-i_{2} \varphi \psi, \\
(\mathrm{j})+(\mathrm{k}) & =\left(i_{2}+i_{1}\right) \varphi \psi-i_{1} \varphi \psi-i_{2} \varphi \psi \\
& =\left(i_{2}+i_{1}\right) s \beta p-i_{1} s \beta p-i_{2} s \beta p \\
& =s\left(\wedge^{2}\left(i_{2}+i_{1}\right)-\wedge^{2} i_{1}-\wedge^{2} i_{2}\right) \beta p \\
& =s \imath(1-T) \eta \beta p .
\end{aligned}
$$

In (k) we apply the naturality of (2.D), and in (l) and (n) the naturality of (2.G).

In (o) we use that given $e_{1} \prec e_{2} \in E_{1}$,

$$
\begin{aligned}
\left(\wedge^{2}\left(i_{2}+i_{1}\right)-\wedge^{2} i_{1}-\wedge^{2} i_{2}\right)\left(e_{1} \wedge e_{2}\right)= & \left(i_{1} e_{1}+i_{2} e_{1}\right) \wedge\left(i_{1} e_{2}+i_{2} e_{2}\right) \\
& -i_{1} e_{1} \wedge i_{1} e_{1}-i_{2} e_{2} \wedge i_{2} e_{2} \\
= & i_{1} e_{1} \wedge i_{2} e_{2}-i_{1} e_{2} \wedge i_{2} e_{1} \\
= & \imath(1-T) \eta\left(e_{1} \wedge e_{2}\right) .
\end{aligned}
$$


Hence by $(\mathrm{m})$ we get

$$
\begin{aligned}
(\mathrm{i}) & =-\omega\left(i_{1} \otimes i_{2}\right) \eta \beta p-\omega\left(i_{2} \otimes i_{1}\right)(\eta \beta-(1-T) \eta \beta) p \\
& =-\omega\left(i_{1} \otimes i_{2}+i_{2} \otimes i_{1} T\right) \eta \beta p .
\end{aligned}
$$

So by (6.C) and (6.D) $\gamma_{2}\{y\}$ is represented by $-\eta \beta(x) \in\left(C_{1} X\right)^{\otimes^{2}}$, and by using also (6.B) and $q \eta=1$ we see that either $-\beta(x)=\alpha(x)-\left(\wedge^{2} d_{2}\right) q(\nabla \psi)(x)$ or $\alpha(x) \in \wedge^{2} C_{1} X$ represents $h_{*}\{x\}$. The proof is now finished.

In the following remark we make some observations about the proof of Theorem 6.1 which will be useful in applications of this result to proper homotopy theory.

Remark 6.2. If we consider diagram (6.B) for the 2 -skeleton $X^{2}$ we get

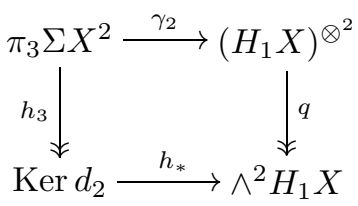

since $\operatorname{Ker} d_{2}=H_{2} X^{2}$. This group is known to be free abelian, hence $h_{3}$ in (6.F) admits a section homomorphism. In fact a concrete section is determined by the homomorphism $r \psi-\omega \eta\left(-\alpha+\left(\wedge^{2} d_{2}\right) q(\nabla \psi)\right) p$ in (6.E). For the definition of this homomorphism we use the homomorphism $\psi$ chosen in the statement of Theorem 6.1, the section $\eta: \wedge^{2} C_{1} X \hookrightarrow\left(C_{1} X\right)^{\otimes^{2}}$ of the natural projection $q$ induced by a total ordering $\preceq$ in the set of 1-cells of $X$, and the homomorphism $\varphi$ induced by the attaching map of 2-cells in $X$. Let us call $\zeta_{X}$ to this section of $h_{3}$. Moreover, in the proof of Theorem 6.1 we check that, if $t_{X}: C_{1} X \rightarrow H_{1} X$ is the natural projection, then the composition of this section with the James-Hopf invariant is $\gamma_{2} \zeta_{X}=\left(t_{X}\right)^{\otimes^{2}} \eta\left(\alpha-\left(\wedge^{2} d_{2}\right) q(\nabla \psi)\right)$. Recall from the statement of Theorem 6.1 that $\alpha$ is the unique homomorphism such that $\varphi \psi=s \alpha p$.

If $Y \subset X$ is a subcomplex, the homomorphism $\varphi^{\prime}$ induced by the attaching map of 2-cells in $Y$ is completely determined by $\varphi$; in fact, $\varphi^{\prime}$ is a restriction of $\varphi$ in the target and the source to the subgroups with basis the cells of $X$ which lie in $Y$. Moreover, the section $\eta$ induces a section $\eta^{\prime}: \wedge^{2} C_{1} Y \hookrightarrow\left(C_{1} Y\right)^{\otimes^{2}}$ which is the section defined by the restriction of the total ordering $\preceq$ to the subset of 1-cells in $Y$. Furthermore, suppose that we choose the basis set $Z_{2}$ of the free abelian group Ker $d_{2}=H_{2} X^{2}$ considered in the statement of Theorem 6.1] in such a way that $Z_{2}$ contains a basis $Z_{2}^{\prime}$ of $H_{2} Y^{2}$, and the homomorphism $\psi$ such that the image of $Z_{2}^{\prime}$ lies in the subgroup generated by the 2-cells of $Y$. In this case there exists a homomorphism $\psi^{\prime}$ from $\left\langle Z_{2}^{\prime}\right\rangle^{\text {nil }}$ to the free nil(2)-group with basis the set of 2cells in $Y$ which is a restriction of $\psi$ in the same way as $\varphi$ restricts to $\varphi^{\prime}$. This homomorphism $\psi^{\prime}$ satisfies the conditions of the statement of Theorem 6.1. Let $\alpha^{\prime}$ be the unique homomorphism such that $\varphi^{\prime} \psi^{\prime}=s \alpha^{\prime} p$, this $\alpha^{\prime}$ is also determined by $\alpha$, in the same way as $\varphi^{\prime}$ and $\psi^{\prime}$, and there is a section of the Hurewicz homomorphism $\zeta_{Y}: H_{2} Y^{2} \hookrightarrow \pi_{3} \Sigma Y^{2}$ induced by $\alpha^{\prime}, \psi^{\prime}$ and $\eta^{\prime}$, as in the case of $\zeta_{X}$. This section $\zeta_{Y}$ is compatible with $\zeta_{X}$ in the sense that the following diagram is commutative:

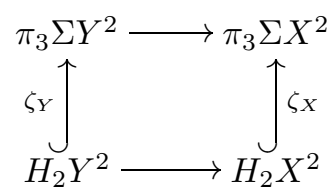


Moreover, we also have that $\gamma_{2} \zeta_{X}^{\prime}=\left(t_{Y}\right)^{\otimes^{2}} \eta^{\prime}\left(\alpha^{\prime}-\left(\wedge^{2} d_{2}\right) q\left(\nabla \psi^{\prime}\right)\right)$, and notice that the homomorphisms $\eta\left(\alpha-\left(\wedge^{2} d_{2}\right) q(\nabla \psi)\right)$ and $\eta^{\prime}\left(\alpha^{\prime}-\left(\wedge^{2} d_{2}\right) q\left(\nabla \psi^{\prime}\right)\right)$ are also compatible, that is, the next diagram commutes:

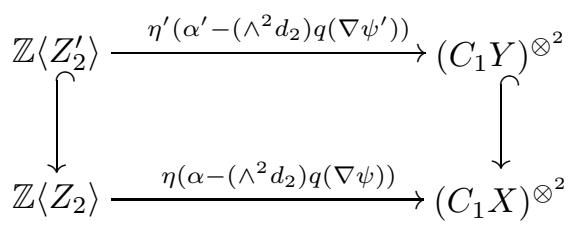

Now we shall give two applications of Theorem 6.1 in group theory, based on the following observation.

Remark 6.3. The image of $h_{*}: H_{2} X \rightarrow \wedge^{2} H_{1} X$ coincides with that of the homomorphism $H_{2} \pi_{1} X \rightarrow H_{2} H_{1} X=\wedge^{2} H_{1} X$ induced by the abelianization $\pi_{1} X \rightarrow$ $\left(\pi_{1} X\right)^{a b}=H_{1} X$, here we use the Hurewicz theorem and the Hopf exact sequence in [13], II.5.2. Hence, by the classical 5 -term exact sequence in the low-dimensional homology of groups, the cokernel of $h_{*}$ is the homomorphism $\tilde{w}: \wedge^{2}\left(\pi_{1} X\right)^{a b} \rightarrow$ $\Gamma_{2} \pi_{1} X / \Gamma_{3} \pi_{1} X$ induced by the commutator bracket. In fact, $\tilde{w}$ is a factorization of $w$ in (2.H) through the natural projection $q$ in (2.A).

A presentation of a group $G$ can be regarded as a pair of homomorphisms

$$
\left\langle E_{2}\right\rangle \stackrel{\varphi_{G}}{\rightarrow}\left\langle E_{1}\right\rangle \stackrel{p_{G}}{\rightarrow} G
$$

such that $\operatorname{Ker} p_{G} \subset\left\langle E_{1}\right\rangle$ is the normal subgroup generated by the image of $\varphi_{G}$. In the next proposition we construct a presentation of $\Gamma_{2} G / \Gamma_{3} G$ as an abelian group from a given presentation of $G$.

Proposition 6.4. Let $G$ be a group presented by (6.I). If $Z_{2}$ is a basis of $\operatorname{Ker} \varphi_{G}^{a b}$, $\psi:\left\langle Z_{2}\right\rangle^{\text {nil }} \rightarrow\left\langle E_{2}\right\rangle^{\text {nil }}$ is a homomorphism such that $\psi^{a b}: \operatorname{Ker} \varphi_{G}^{a b} \hookrightarrow \mathbb{Z}\left\langle E_{2}\right\rangle$ is the inclusion, and $\alpha: \mathbb{Z}\left\langle Z_{2}\right\rangle \rightarrow \wedge^{2} \mathbb{Z}\left\langle E_{1}\right\rangle$ satisfies $s \alpha p=\varphi_{G}^{\text {nil }} \psi$, then the following sequence is exact:

$$
\mathbb{Z}\left\langle Z_{2}\right\rangle \oplus \mathbb{Z}\left\langle E_{2}\right\rangle \otimes \mathbb{Z}\left\langle E_{1}\right\rangle \oplus \wedge^{2} \mathbb{Z}\left\langle E_{2}\right\rangle \stackrel{\zeta}{\longrightarrow} \wedge^{2} \mathbb{Z}\left\langle E_{1}\right\rangle \stackrel{\tilde{w}\left(\wedge^{2} p_{G}^{a b}\right)}{\rightarrow} \Gamma_{2} G / \Gamma_{3} G .
$$

Here $\zeta=\left(\alpha, q\left(\varphi_{G}^{a b} \otimes 1\right), \wedge^{2} \varphi_{G}^{a b}\right)$.

Proof. If we take $X$ to be the 2-dimensional $C W$-complex with the attaching map of 2-cells given by $\varphi_{G}$, then, by Theorem 6.1 and Remark 6.3 we get an exact sequence

$$
\mathbb{Z}\left\langle Z_{2}\right\rangle \stackrel{\left(\wedge^{2} p_{G}^{a b}\right) \alpha}{\longrightarrow} \wedge^{2} G^{a b} \stackrel{\tilde{w}}{\rightarrow} \Gamma_{2} G / \Gamma_{3} G
$$

Therefore this proposition follows by (2.F).

The kernel of $\tilde{w}$ in 6 .J is the second homology group of $G^{n i l}$ in the variety of groups of nilpotency degree 2, as defined in [17]; see Theorem 5.1 in [11. This abelian group is denoted by $\mathfrak{B}_{1}\left(G^{\text {nil }}, \mathbb{Z}\right)$ in [17] and $H_{2}^{N i l} G^{\text {nil }}$ in [11]. Thus if

$$
\left(\begin{array}{c}
\beta_{1} \\
\beta_{2} \\
\beta_{3}
\end{array}\right): \mathbb{Z}\langle F\rangle \hookrightarrow \mathbb{Z}\left\langle Z_{2}\right\rangle \oplus \mathbb{Z}\left\langle E_{2}\right\rangle \otimes \mathbb{Z}\left\langle E_{1}\right\rangle \oplus \wedge^{2} \mathbb{Z}\left\langle E_{2}\right\rangle
$$


is the kernel of $\zeta$, then by (2.F) one easily checks that

Corollary 6.5. There is an exact sequence

$$
\mathbb{Z}\langle F\rangle \stackrel{\beta_{1}}{\longrightarrow} \mathbb{Z}\left\langle Z_{2}\right\rangle \rightarrow H_{2}^{N i l} G^{\text {nil }} .
$$

This is in fact an abelian presentation of the homology group $H_{2}^{N i l} G^{\text {nil }}$. This presentation and the universal coefficient exact sequences for $H_{*}^{N i l}$ in 11 yield an explicit computation of $H_{n}^{N i l} G^{\text {nil }}$ for any finitely presented group $G$ and $n \geq 1$.

\section{Commutative CO-H-Structures on Degree 2 Moore spaces}

A Moore space of type $(A, n)$ is a simply connected space $M=M(A, n)$ whose unique non-trivial reduced homology group is $H_{n} M=A$, this space is unique up to homotopy type. The number $n$ is the degree of the Moore space. The quadratic complex $\sigma_{A}$ of the Moore space $M(A, 2)$ is the suspension of the quadratic complex concentrated in degrees 1 and 2 whose nil(2)-module has as a basis a homomorphism

$$
\bar{\varphi}_{A}:\left\langle A_{2}\right\rangle \rightarrow\left\langle A_{1}\right\rangle
$$

such that $\bar{\varphi}_{A}^{a b}$ is injective and Coker $\bar{\varphi}_{A}^{a b}=A$, the natural projection is denoted by

$$
\hat{p}_{A}: \mathbb{Z}\left\langle A_{1}\right\rangle \rightarrow A \text {. }
$$

Hence, by Lemma 4.6, $\sigma_{A}$ is the reduced quadratic complex concentrated in degrees 2 and 3 such that the basis of its reduced quadratic module is $-\varphi_{A}$, where $\varphi_{A}=$ $\bar{\varphi}_{A}^{n i l}$. This suspended quadratic complex is constructed as in (3.C) and carries a canonical cogroup structure $\mu_{A}, \nu_{A}$, which is determined by $\varphi_{A}$; see Theorem 5.6 and Corollary 5.8

The goal of this section is constructing and computing an extension element

$$
c_{A} \in \operatorname{Ext}\left(A, S^{2} A\right)
$$

such that $c_{A}=0$ if and only if $M(A, 2)$ admits a commutative co-H-structure. We shall see that this element is natural with respect to homomorphisms $f: A \rightarrow B$, that is,

$$
\left(S^{2} f\right)_{*} c_{A}=f^{*} c_{B} \in \operatorname{Ext}\left(A, S^{2} B\right)
$$

therefore it represents an element in the cohomology of the category $\mathbf{A b}$ of abelian groups with coefficients in the bifunctor $\operatorname{Ext}\left(-, S^{2}\right)$ (see for example [7, VI.1),

$$
c \in H^{0}\left(\mathbf{A b}, \operatorname{Ext}\left(-, S^{2}\right)\right) \text {. }
$$

Moreover, we shall show that $c$ is a non-trivial element of order 2 .

Since Moore spaces of degree 2 are 3-dimensional, the homotopy category of these spaces coincides with that of their quadratic complexes. There is a wellknown natural central extension

$$
\operatorname{Ext}(A, \Gamma B) \stackrel{j}{\hookrightarrow}\left[\sigma_{A}, \sigma_{B}\right] \stackrel{H_{2}}{\rightarrow} \operatorname{Hom}(A, B) .
$$

Here $H_{2}=\pi_{2}$ as defined in (4.A), by the Hurewicz theorem. Moreover, given an element $\beta \in \operatorname{Ext}(A, \Gamma B)$ represented by $\bar{\beta}: \mathbb{Z}\left\langle A_{2}\right\rangle \rightarrow \Gamma B, j \beta$ is the morphism of quadratic complexes with $(j \beta)_{n}=0$ if $n \neq 3$,

$$
(j \beta)_{3} r=\bar{\beta} p:\left\langle A_{2}\right\rangle^{n i l} \rightarrow \Gamma B=\pi_{3} M(B, 2)=\operatorname{Ker} d_{3} \subset\left(\sigma_{B}\right)_{3},
$$


and $(j \beta)_{3} \omega=0$. The natural extension (7.B) satisfies the following linear distributivity law (see [8]): given two morphisms $\sigma_{A} \stackrel{f}{\rightarrow} \sigma_{B} \stackrel{g}{\rightarrow} \sigma_{C}$ and elements $\beta \in \operatorname{Ext}(A, \Gamma B), \zeta \in \operatorname{Ext}(B, \Gamma C)$, then

$$
(g+j \zeta)(f+j \beta)=g f+j\left(\Gamma H_{2} g\right)_{*} \beta+j\left(H_{2} f\right)^{*} \zeta \in\left[\sigma_{A}, \sigma_{C}\right] .
$$

Remark 7.1. In this section we often identify a morphism in $\mathbf{Q}$ with its homotopy class in $\mathbf{Q} / \simeq$, hence the symbol "=" sometimes means that two homomorphisms in $\mathbf{Q}$ are homotopic, but may not be strictly equal. However, it will be clear from the context when "=" stands for equality and when for homotopy.

If we combine (7.B) with (2.C), we easily check that the following sequence, where $p_{1}=(1,0)$ and $p_{2}=(0,1)$, is a central extension as well:

$$
\operatorname{Ext}\left(A, B^{\otimes^{2}}\right) \stackrel{j l_{*}}{\longrightarrow}\left[\sigma_{A}, \sigma_{B} \vee \sigma_{B}\right] \stackrel{\left(p_{1_{*}}, p_{2_{*}}\right)}{\longrightarrow}\left[\sigma_{A}, \sigma_{B}\right] \times\left[\sigma_{A}, \sigma_{B}\right] .
$$

Here if $\beta \in \operatorname{Ext}\left(A, B^{\otimes^{2}}\right)$ is represented by $\left(\hat{p}_{B} \otimes \hat{p}_{B}\right) \tilde{\beta}$ for some $\tilde{\beta}: \mathbb{Z}\left\langle A_{2}\right\rangle \rightarrow$ $\mathbb{Z}\left\langle B_{1}\right\rangle^{\otimes^{2}}$, then $j l_{*} \beta$ is the morphism with $\left(j l_{*} \beta\right)_{n}=0$ for $n \neq 3$ and

$$
\left(j l_{*} \beta\right)_{3} r=\omega\left(i_{1} \otimes i_{2}+i_{2} \otimes i_{1} T\right) \tilde{\beta} p:\left\langle A_{2}\right\rangle^{n i l} \rightarrow\left(\sigma_{B} \vee \sigma_{B}\right)_{3} .
$$

Here we use (6.D) and the well-known fact that the Whitehead product $\left[i_{1 *}, i_{2 *}\right]$ : $\pi_{2} M \otimes \pi_{2} M \rightarrow \pi_{3}(M \vee M)$ coincides with $l$ in (2.D) for a Moore space $M=M(B, 2)$.

From (7.D) it is easy to observe that there is an effective and transitive action of $\operatorname{Ext}\left(A, A^{\otimes^{2}}\right)$ in the set of homotopy classes of co-H-multiplications for $M(A, 2)$; see [2]. Recall that this set is formed by the elements $\mu \in\left[\sigma_{A}, \sigma_{A} \vee \sigma_{A}\right]$ such that $(1,0) \mu=(0,1) \mu=1$. The homotopy class of $\mu_{A}$ is such a co-H-multiplication and any other co-H-multiplication verifies $\mu=\mu_{A}+j l_{*} \beta$ for a unique $\beta \in \operatorname{Ext}\left(A, A^{\otimes^{2}}\right)$, in particular, there is a unique $\hat{c}_{A} \in \operatorname{Ext}\left(A, A^{\otimes^{2}}\right)$ such that $\mu_{A}+j l_{*} \hat{c}_{A}=\left(i_{2}, i_{1}\right) \mu_{A}$. By using the canonical morphism $q^{\prime}$ in (2.B) we get an element

$$
c_{A}=q_{*}^{\prime} \hat{c}_{A} \in \operatorname{Ext}\left(A, S^{2} A\right)
$$

which satisfies the following obstruction property.

Proposition 7.2. The element $c_{A} \in \operatorname{Ext}\left(A, S^{2} A\right)$ is zero if and only if $M(A, 2)$ admits a commutative co-H-multiplication. Moreover, it is natural in the category of abelian groups, in the sense of (7.A).

Recall that a co-H-multiplication $\mu$ is commutative if $\mu$ is homotopic to $\left(i_{2}, i_{1}\right) \mu$.

Proof of Proposition 7.2. As we have seen, $M(A, 2)$ admits a commutative co-Hmultiplication if and only if there exists $\beta \in \operatorname{Ext}\left(A, A^{\otimes^{2}}\right)$ such that

$$
\begin{aligned}
\mu_{A}+j l_{*} \beta & =\left(i_{2}, i_{1}\right)\left(\mu_{A}+j l_{*} \beta\right) \\
& =\left(i_{2}, i_{1}\right) \mu_{A}+j\left(\Gamma\left(i_{2}, i_{1}\right)\right)_{*} l_{*} \beta \\
& =\left(i_{2}, i_{1}\right) \mu_{A}+j l_{*} T_{*} \beta .
\end{aligned}
$$

In (a) we use (7.C) and in (b) (2.E). Therefore $\hat{c}_{A}=(1-T)_{*} \beta$ and, by the construction of $S^{2}$ in (2.B), we get $c_{A}=q_{*}^{\prime} \hat{c}_{A}=q_{*}^{\prime}(1-T)_{*} \beta=0$.

On the other hand, the functor $\operatorname{Ext}(A,-)$ is known to be right-exact, so that $0=c_{A}=q_{*}^{\prime} \hat{c}_{A}$ if and only if there exists $\beta \in \operatorname{Ext}\left(A, A^{\otimes^{2}}\right)$ such that $\hat{c}_{A}=(1-T)_{*} \beta$; see (2.B). In this case one can check as above that $\mu_{A}+j l_{*} \beta$ is a commutative co-H-multiplication for $M(A, 2)$. 
Now we prove the naturality of $c_{A}$. Given a homomorphism $f: A \rightarrow B$ we can choose a morphism $F: \sigma_{A} \rightarrow \sigma_{B}$ such that $H_{2} F=f$, then

$$
\begin{aligned}
\left(i_{2}, i_{1}\right)(F \vee F) \mu_{A} & =(F \vee F)\left(i_{2}, i_{1}\right) \mu_{A} \\
& =(F \vee F)\left(\mu_{A}+j l_{*} \hat{c}_{A}\right) \\
& =(F \vee F) \mu_{A}+j(\Gamma(f \oplus f))_{*} l_{*} \hat{c}_{A} \\
& =(F \vee F) \mu_{A}+j l_{*}(f \otimes f)_{*} \hat{c}_{A}, \\
\left(i_{2}, i_{1}\right) \mu_{B} F & =\left(\mu_{B}+j l_{*} \hat{c}_{B}\right) F \\
& =\mu_{B} F+j l_{*} f^{*} \hat{c}_{B}
\end{aligned}
$$

In (c) and (e) we apply (7.C), and in (d) (2.E). Hence

(f) $\left(i_{2}, i_{1}\right)\left((F \vee F) \mu_{A}-\mu_{B} F\right)=(F \vee F) \mu_{A}-\mu_{B} F+j l_{*}(f \otimes f)_{*} \hat{c}_{A}-j l_{*} f^{*} \hat{c}_{B}$.

By (1.D) there exists a unique $\zeta \in \operatorname{Ext}\left(A, B^{\otimes^{2}}\right)$ such that $\mu_{B} F+j l_{*} \zeta=(F \vee F) \mu_{A}$, therefore by (7.C) and (2.E) $\left(i_{2}, i_{1}\right) \mu_{B} F+j l_{*} T_{*} \zeta=\left(i_{2}, i_{1}\right)(F \vee F) \mu_{A}$, and hence (I) is equivalent to the following equality in $\operatorname{Ext}\left(A, B^{\otimes^{2}}\right)$ :

$$
T_{*} \zeta=\zeta+(f \otimes f)_{*} \hat{c}_{A}-f^{*} \hat{c}_{B}
$$

Thus

$$
f^{*} c_{B}-\left(S^{2} f\right)_{*} c_{A}=q_{*}^{\prime}\left(f^{*} \hat{c}_{B}-(f \otimes f)_{*} \hat{c}_{A}\right)=q_{*}^{\prime}(1-T)_{*} \zeta=0 .
$$

Here we use the naturality and exactness of (2.B) . The proof is now finished.

Corollary 7.3. The extension element $c_{A} \in \operatorname{Ext}\left(A, S^{2} A\right)$ does not depend on the choice of the homomorphism $\bar{\varphi}_{A}$ for the construction of $\sigma_{A}$ and $\mu_{A}$.

Proof. Take $\bar{\varphi}_{A}^{\prime}$ in the same conditions as $\bar{\varphi}_{A}$. Let $\sigma_{A}^{\prime}$ be the Moore space quadratic complex of type $(A, 2)$ constructed by using $\bar{\varphi}_{A}^{\prime}$ instead of $\bar{\varphi}_{A}$, and $c_{A}^{\prime} \in$ $\operatorname{Ext}\left(A, S^{2} A\right)$ the extension element related to $\bar{\varphi}_{A}^{\prime}$, constructed in the same way as $c_{A}$. Choose $B=A, f: A \rightarrow B$ the identity homomorphism $f=1_{A}$, and $F: \sigma_{A} \rightarrow \sigma_{A}^{\prime}$ a quadratic complex morphism inducing $1_{A}$ in $H_{2}$. Then the same technique used in Proposition 7.2 to prove the naturality of $c_{A}$ shows that $c_{A}=$ $c_{A}^{\prime} \in \operatorname{Ext}\left(A, S^{2} A\right)$.

Corollary 7.4. For any abelian group $2 c_{A}=0$. Therefore if $\operatorname{Ext}\left(A, S^{2} A\right)$ has no element of order 2 , then $M(A, 2)$ admits a commutative co-H-multiplication.

Proof. In general $S^{2}\left(n 1_{A}\right)=n^{2} 1_{S^{2} A}$, then by the naturality property of $c_{A}$ in (7.A),

$$
0=\left(S^{2}\left(1_{A}+1_{A}\right)\right)_{*} c_{A}-\left(1_{A}+1_{A}\right)^{*} c_{A}=4 c_{A}-2 c_{A}=2 c_{A} .
$$

In the next theorem we compute a formula for $c_{A}$ from a free resolution of $A$.

Theorem 7.5. If $\mathbb{Z}\left\langle A_{2}\right\rangle \stackrel{\psi_{A}}{\hookrightarrow} \mathbb{Z}\left\langle A_{1}\right\rangle \stackrel{\hat{p}_{A}}{\rightarrow} A$ is a free resolution of $A$ such that $\psi_{A}(b)=$ $\sum_{i=1}^{n} \epsilon_{i} a_{i}$ for $b \in A_{2}, a_{i} \in A_{1}$ and $\epsilon_{i} \in \mathbb{Z}$, then the extension element $c_{A} \in$ $\operatorname{Ext}\left(A, S^{2} A\right)$ is represented by the homomorphism $\check{c}_{A}: \mathbb{Z}\left\langle A_{2}\right\rangle \rightarrow S^{2} A$ defined by

$$
\check{c}_{A}(b)=\sum_{i=1}^{n}\left(\begin{array}{c}
\left|\epsilon_{i}\right| \\
2
\end{array}\right) \hat{p}_{A}\left(a_{i}\right)^{2} .
$$


Proof. We can take the basis $-\varphi_{A}=-\varphi \in$ nil of the reduced quadratic complex $\sigma_{A}$ of the Moore space $M(A, 2)$ to be defined by $\varphi(b)=\epsilon_{1} a_{1}+\cdots+\epsilon_{n} a_{n} \in\left\langle A_{1}\right\rangle^{\text {nil }}$, hence $\varphi^{a b}=\psi_{A}$. The following equalities hold:

(a)

$$
\begin{aligned}
\left(\mu_{\sigma}-\left(i_{2}, i_{1}\right) \mu_{\sigma}\right)_{2} & =i_{1}+i_{2}-i_{1}-i_{2} \\
& =\left[i_{1}, i_{2}\right] \\
& =s \imath \Delta p \\
& =s q\left(i_{1} \otimes i_{2}\right) \Delta p \\
& =d_{3} \omega\left(i_{1} \otimes i_{2}\right) \Delta p .
\end{aligned}
$$

Here we use Theorem 5.6]in (a), Lemma 5.10 in (b), the definition of $\imath$ (2.A) in (c), and (3.C) in (d). Hence the homomorphism $\left(i_{1} \otimes i_{2}\right) \Delta: \mathbb{Z}\left\langle A_{1}\right\rangle \rightarrow\left(\mathbb{Z}\left\langle A_{1}\right\rangle \oplus \mathbb{Z}\left\langle A_{1}\right\rangle\right)^{\otimes^{2}}$ determines a 0-homotopy between $\mu_{\sigma}-\left(i_{2}, i_{1}\right) \mu_{\sigma}$ and $f: \Sigma \sigma \rightarrow \Sigma \sigma \vee \Sigma \sigma$ with $f_{n}=0$ for $n \neq 3, f_{3} \omega=0$ and

$$
f_{3} r=\left(\mu_{\sigma}-\left(i_{2}, i_{1}\right) \mu_{\sigma}\right)_{3} r-\omega\left(i_{1} \otimes i_{2}\right) \Delta p(-\varphi) .
$$

Moreover,

$$
\begin{aligned}
r\left[i_{1}, i_{2}\right] & =r s q\left(i_{1} \otimes i_{2}\right) \Delta p \\
& =\omega\left(\varphi^{a b} \oplus \varphi^{a b} \otimes^{\otimes^{2}}\left(i_{1} \otimes i_{2}\right) \Delta p\right. \\
& =\omega\left(i_{1} \otimes i_{2}\right)\left(\varphi^{a b} \otimes \varphi^{a b}\right) \Delta p \\
\left(\left(i_{2}, i_{1}\right) \mu_{\sigma}\right)_{3} r & =r\left(i_{2}+i_{1}\right)+\omega\left(i_{1} \otimes i_{2}\right)\left(\nabla \varphi+\Delta \varphi^{a b}\right) p .
\end{aligned}
$$

Here we use (b) and (c) in (f), (3.C) in (g), and Theorem 5.6 in (h). Hence by Theorem 5.6 and Corollary 5.7

$$
\begin{aligned}
\left(-\left(i_{2}, i_{1}\right) \mu_{\sigma}\right)_{3} r= & -r\left(i_{2}+i_{1}\right)-\omega\left(i_{1} \otimes i_{2}\right)\left(\nabla \varphi+\Delta \varphi^{a b}\right) p \\
& +\omega\left(i_{1}+i_{2}\right)^{\otimes^{2}}\left(\nabla \varphi+\Delta \varphi^{a b}\right) p
\end{aligned}
$$

and

$$
\begin{aligned}
(\mathrm{e})= & r\left(i_{1}+i_{2}\right)+\omega\left(i_{2} \otimes i_{1}\right)\left(\nabla \varphi+\Delta \varphi^{a b}\right) p \\
& -r\left(i_{2}+i_{1}\right)-\omega\left(i_{1} \otimes i_{2}\right)\left(\nabla \varphi+\Delta \varphi^{a b}\right) p \\
& +\omega\left(i_{1}+i_{2}\right)^{2}\left(\nabla \varphi+\Delta \varphi^{a b}\right) p \\
& +\omega\left(\left(i_{1}+i_{2}\right) \otimes\left(-i_{1}-i_{2}\right)\right)\left(\nabla \varphi+\Delta \varphi^{a b}\right) p-\omega\left(i_{1} \otimes i_{2}\right) \Delta\left(-\varphi^{a b}\right) p \\
= & r\left[i_{1}, i_{2}\right]-\omega\left(i_{1} \otimes i_{2}\right)\left(\nabla \varphi+\Delta \varphi^{a b}+\Delta\left(-\varphi^{a b}\right)\right) p \\
& +\omega\left(i_{2} \otimes i_{1}\right)\left(\nabla \varphi+\Delta \varphi^{a b}\right) p \\
= & \omega\left(i_{1} \otimes i_{2}\right)\left(\left(\varphi^{a b} \otimes \varphi^{a b}\right) \Delta-\nabla \varphi\right)+\omega\left(i_{2} \otimes i_{1}\right)\left(\nabla \varphi+\Delta \varphi^{a b}\right) .
\end{aligned}
$$

In $(\mathrm{j})$ we use that $\omega$ is central, and in $(\mathrm{k})$ we apply $(\mathrm{f})$.

By using Lemma [5.9, one can prove the equality $T \nabla(-\varphi)=\nabla \varphi+\Delta \varphi^{a b}$ by an easy computation, therefore by Lemma 5.10

$$
T\left(\nabla \varphi+\Delta \varphi^{a b}\right)=\left(\varphi^{a b} \otimes \varphi^{a b}\right) \Delta-\nabla \varphi,
$$

and hence by (i)

$$
f_{3} r=\omega\left(i_{1} \otimes i_{2}+i_{2} \otimes i_{1} T\right) T\left(\nabla \varphi+\Delta \varphi^{a b}\right) p .
$$

So, by (7.E), $\hat{c}_{A} \in \operatorname{Ext}\left(A, A^{\otimes^{2}}\right)$ is represented by $-\left(\hat{p}_{A} \otimes \hat{p}_{A}\right) T\left(\nabla \varphi+\Delta \varphi^{a b}\right)$, and then $c_{A} \in \operatorname{Ext}\left(A, S^{2} A\right)$ is represented by

$$
-q^{\prime}\left(\hat{p}_{A} \otimes \hat{p}_{A}\right) T\left(\nabla \varphi+\Delta \varphi^{a b}\right)=-\left(S^{2} \hat{p}_{A}\right) q^{\prime} T\left(\nabla \varphi+\Delta \varphi^{a b}\right)=-\left(S^{2} \hat{p}_{A}\right)\left(\nabla \varphi+\Delta \varphi^{a b}\right) .
$$


Here we use the naturality of $q^{\prime}$ and the fact that $q^{\prime} T=q^{\prime}$; see (2.B).

From these computations we see that we can take $\check{c}_{A}=-\left(S^{2} \hat{p}_{A}\right)\left(\nabla \varphi+\Delta \varphi^{a b}\right)$. Let us check now that $\check{c}_{A}$ satisfies the formula of the statement. The first equality in the next formula is a consequence of Lemma 5.9, in the third we apply the fact that $\hat{p}_{A} \psi_{A}=0$

$$
\begin{aligned}
& -\check{c}_{A}(b)=\sum_{1 \leq i<j \leq n} \epsilon_{i} \epsilon_{j} \hat{p}_{A}\left(a_{i}\right) \hat{p}_{A}\left(a_{j}\right)+\sum_{\epsilon_{i}>0} \frac{\epsilon_{i}\left(\epsilon_{i}+1\right)}{2} \hat{p}_{A}\left(a_{i}\right)^{2} \\
& +\sum_{\epsilon_{i}<0} \frac{\epsilon_{i}\left(\epsilon_{i}-1\right)}{2} \hat{p}_{A}\left(a_{i}\right)^{2} \\
& =\sum_{0<\epsilon_{i} \text { even }}\left[\left(\epsilon_{i} \hat{p}_{A}\left(a_{i}\right)\right)\left(\frac{\epsilon_{i}}{2} \hat{p}_{A}\left(a_{i}\right)+\sum_{j \neq i} \epsilon_{j} \hat{p}_{A}\left(a_{j}\right)\right)+\frac{\epsilon_{i}}{2} \hat{p}_{A}\left(a_{i}\right)^{2}\right] \\
& +\sum_{0<\epsilon_{i} \text { odd }}\left(\epsilon_{i} \hat{p}_{A}\left(a_{i}\right)\right)\left(\frac{\epsilon_{i}+1}{2} \hat{p}_{A}\left(a_{i}\right)+\sum_{j \neq i} \epsilon_{j} \hat{p}_{A}\left(a_{j}\right)\right) \\
& +\sum_{0>\epsilon_{i} \text { even }}\left[\left(\epsilon_{i} \hat{p}_{A}\left(a_{i}\right)\right)\left(\frac{\epsilon_{i}}{2} \hat{p}_{A}\left(a_{i}\right)+\sum_{j \neq i} \epsilon_{j} \hat{p}_{A}\left(a_{j}\right)\right)-\frac{\epsilon_{i}}{2} \hat{p}_{A}\left(a_{i}\right)^{2}\right] \\
& +\sum_{0>\epsilon_{i} \text { odd }}\left(\epsilon_{i} \hat{p}_{A}\left(a_{i}\right)\right)\left(-\frac{\epsilon_{i}+1}{2} \hat{p}_{A}\left(a_{i}\right)+\sum_{j \neq i} \epsilon_{j} \hat{p}_{A}\left(a_{j}\right)\right) \\
& =\sum_{0<\epsilon_{i} \text { even }}\left[\left(\epsilon_{i} \hat{p}_{A}\left(a_{i}\right)\right)\left(-\frac{\epsilon_{i}}{2} \hat{p}_{A}\left(a_{i}\right)\right)+\frac{\epsilon_{i}}{2} \hat{p}_{A}\left(a_{i}\right)^{2}\right] \\
& +\sum_{0<\epsilon_{i} \text { odd }}\left(\epsilon_{i} \hat{p}_{A}\left(a_{i}\right)\right)\left(-\frac{\epsilon_{i}-1}{2} \hat{p}_{A}\left(a_{i}\right)\right) \\
& +\sum_{0>\epsilon_{i} \text { even }}\left[\left(\epsilon_{i} \hat{p}_{A}\left(a_{i}\right)\right)\left(-\frac{\epsilon_{i}}{2} \hat{p}_{A}\left(a_{i}\right)\right)-\frac{\epsilon_{i}}{2} \hat{p}_{A}\left(a_{i}\right)^{2}\right] \\
& +\sum_{0>\epsilon_{i} \text { odd }}\left(\epsilon_{i} \hat{p}_{A}\left(a_{i}\right)\right)\left(-\frac{\epsilon_{i}+1}{2} \hat{p}_{A}\left(a_{i}\right)\right) \\
& =\sum_{\epsilon_{i}>0}-\frac{\epsilon_{i}^{2}-\epsilon_{i}}{2} \hat{p}_{A}\left(a_{i}\right)^{2}+\sum_{\epsilon_{i}<0}-\frac{\epsilon_{i}^{2}+\epsilon_{i}}{2} \hat{p}_{A}\left(a_{i}\right)^{2} \\
& =-\sum_{i=1}^{n}\left(\begin{array}{c}
\left|\epsilon_{i}\right| \\
2
\end{array}\right) \hat{p}_{A}\left(a_{i}\right)^{2} \text {. }
\end{aligned}
$$

Corollary 7.6. If $A$ is a direct sum of cyclic groups, then $M(A, 2)$ admits a commutative co-H-multiplication if and only if $A$ has no elements of order 2.

Proof. Suppose that $A=\bigoplus_{i \in I} \mathbb{Z} / n_{i}$ where $n_{i} \in \mathbb{Z}$ is either 0 or a prime power. We take the following free resolution of $A$ :

$$
\mathbb{Z}\left\langle\left\{a_{2, i} ; \quad i \in I, n_{i} \neq 0\right\}\right\rangle \stackrel{\psi_{A}}{\longrightarrow} \mathbb{Z}\left\langle\left\{a_{1, i} ; \quad i \in I\right\}\right\rangle \stackrel{\hat{p}_{A}}{\rightarrow} A,
$$

here $\psi_{A}\left(a_{2, i}\right)=n_{i} a_{1, i}$ and $\hat{p}_{A}\left(a_{1, i}\right)$ is the generator of the direct summand $\mathbb{Z} / n_{i} \subset$ $A$. If we choose a total ordering $\preceq$ in $I$ the symmetric square of $A$ is $S^{2} A=$ 
$\bigoplus_{i \preceq j} \mathbb{Z} /\left(n_{i}, n_{j}\right)$, where $\left(n_{i}, n_{j}\right)$ is the greatest common divisor and the direct summand $\mathbb{Z} /\left(n_{i}, n_{j}\right)$ is generated by $\hat{p}_{A}\left(a_{1, i}\right) \hat{p}_{A}\left(a_{1, j}\right)$. The element $c_{A} \in \operatorname{Ext}\left(A, S^{2} A\right)$ is represented by the homomorphism $\check{c}_{A}$ with $\check{c}_{A}\left(a_{2, i}\right)=\left(\begin{array}{c}n_{i} \\ 2\end{array}\right) \hat{p}_{A}\left(a_{1, i}\right)^{2}$. Therefore, if $n_{i}$ is odd, then

$$
\check{c}_{A}\left(a_{2, i}\right)=\left(n_{i} \hat{p}_{A}\left(a_{1, i}\right)\right)\left(\frac{n_{i}-1}{2} \hat{p}_{A}\left(a_{1, i}\right)\right)=0 ;
$$

otherwise $n_{i}=2^{m}$ and $\check{c}_{A}\left(a_{2, i}\right)=2^{m-1} \hat{p}_{A}\left(a_{i i}\right)^{2}$ is the unique element of order 2 in $\mathbb{Z} / 2^{m}=\mathbb{Z} /\left(n_{i}, n_{i}\right) \subset S^{2} A$. If $A$ has no elements of order 2 , then $n_{i}$ is always odd or zero, and $\check{c}_{A}=0$ is the trivial homomorphism, hence $c_{A}=0$. On the other hand, if $A$ has order 2 elements, then $n_{i}=2^{m}$ for some $i \in I$, and the usual computation of $\operatorname{Ext}\left(\mathbb{Z}_{2^{m}}, \mathbb{Z}_{2^{m}}\right) \simeq \mathbb{Z}_{2^{m}}$ shows that $c_{A} \neq 0$.

Remark 7.7. Corollary 7.6 is no longer true if $A$ is not a direct sum of cyclic groups. Consider $A=\mathbb{Q} / \mathbb{Z}$. It is well known that $(\mathbb{Q} / \mathbb{Z})^{\otimes^{2}}=0$, hence $S^{2}(\mathbb{Q} / \mathbb{Z})=0$ and $\operatorname{Ext}\left(A, S^{2} A\right)=0$, in particular, $c_{A}=0$. But the class of $1 / 2$ in $\mathbb{Q} / \mathbb{Z}$ is a non-trivial element of order 2 .

\section{ACKNOWLEDGEMENT}

Most of this work was written during a stay at the Department of Algebra and Geometry of the University of Barcelona in 2002, invited by C. Casacuberta, to whom I am grateful for his hospitality.

\section{REFERENCES}

1. M. Aguilar, S. Gitler, and C. Prieto, Algebraic Topology from a Homotopical Viewpoint, Springer-Verlag, New York, 2002. MR 2003c:55001

2. M. Arkowitz and M. Golasiński, Co-H-structures on Moore spaces of type (G,2), Canad. J. Math. 46 (1994), no. 4, 673-686. MF 95e:55012

3. Z. Arvasi, Crossed squares and 2-crossed modules of commutative algebras, Theory Appl. Categ. 3 (1997), no. 7, 160-181. MR.98i:18006

4. R. Ayala, M. Cárdenas, F. Muro, and A. Quintero, An elementary approach to the projective dimension in proper homotopy theory, Comm. in Alg. 31 (2003), no. 12, 5995-6017.

5. R. Ayala, E. Domínguez, A. Márquez, and A. Quintero, Moore spaces in proper homotopy, Tsukuba J. Math. 19 (1995), 305-327. MR96k:55015

6. H.-J. Baues, Algebraic Homotopy, Cambridge University Press, 1989. MR90i:55016

7. Combinatorial Homotopy and 4-Dimensional Complexes, Walter de Gruyter, Berlin, 1991. MR92h:55008

8. _ Homotopy Type and Homology, Oxford University Press, 1996. MR 97f:55001

9. H.-J. Baues and D. Conduché, The central series for Peiffer commutators in groups with operators, J. Algebra 133 (1990), 1-34. MR91m:20051

10. H.-J. Baues, M. Hartl, and T. Pirashvili, Quadratic categories and square rings, J. Pure Appl. Algebra 122 (1997), 1-40. MR99f:18017

11. H.-J. Baues and T. Pirashvili, A universal coefficient theorem for quadratic functors, J. Pure Appl. Algebra 148 (2000), no. 1, 1-15. MF2001h:18012

12. A. L. Blakers and W. S. Massey, Products in homotopy theory, Annals of Mathematics 58 (1953), no. 2, 295-324. MR15:731f

13. K. S. Brown, Cohomology of Groups, Springer-Verlag, New York, 1982. MR83k:20002

14. R. Brown and M. Golasiński, A model structure for the homotopy theory of crossed complexes, Cahiers Topologie Géom. Différentielle Catég. 30 (1989), no. 1, 61-82. MF 90f:18012

15. R. Brown and J.-L. Loday, Van Kampen theorems for diagrams of spaces, Topology 26 (1987), no. 3, 311-335. MR88m:55008

16. D. Conduché, Modules croisés généralisés de longueur 2, J. Pure Appl. Algebra 34 (1984), no. 2-3, 155-178. MR86g:20068 
17. C. R. Leedham-Green, Homology in varieties of groups I, Trans. Amer. Math. Soc. 162 (1971), 1-14. MR 44:1735

18. S. Mac Lane, Cohomology theory in abstract groups III, Ann. of Math. 50 (1949), 736-761. MR11:415f

19. S. Mac Lane and J. H. C. Whitehead, On the 3-type of a complex, Proc. Nat. Acad. Sci. 36 (1950), 41-48. MR11:450h

20. F. Muro, On the proper homotopy classification of locally compact $A_{n}^{2}$-polyhedra, In preparation.

21. T. Mutlu and T. Porter, Freeness conditions for 2-crossed modules and complexes, Theory Appl. Categ. 4 (1998), no. 8, 174-194. MR99j:18013

22. R. Peiffer, Über Identitäten zwischen Relationen, Math. Ann. 121 (1949), 67-99. MR 11:322c

23. J. H. C. Whitehead, Combinatorial homotopy II, Bull. Amer. Math. Soc. 55 (1949), 453-496. MR 11:48c

24. — A certain exact sequence, Ann. Math. 52 (1950), 51-110. MR12:43c

Departamento de Geometría y Topología, Universidad de Sevilla, Apartado de Correos 1160, 41080 Sevilla, Spain

E-mail address: fmuro@us.es 